Mathematical Programming manuscript No.

(will be inserted by the editor)

Stephen J. Wright

\title{
On the Convergence of the Newton/Log-Barrier Method
}

Received: date / Revised version: date

\begin{abstract}
In the Newton/log-barrier method, Newton steps are taken for the log-barrier function for a fixed value of the barrier parameter until a certain convergence criterion is satisfied The barrier parameter is then decreased and the Newton process is repeated. A naive analysis indicates that Newton's method does not exhibit superlinear convergence to the minimizer of each instance of the log-barrier function until it reaches a very small neighborhood of the minimizer. By partitioning according to the subspace of active constraint gradients, however, we show that this neighborhood is actually quite large, thus explaining why reasonably fast local convergence can be attained in practice. Moreover, we show that the overall convergence rate of the Newton/log-barrier algorithm is superlinear in the number of function/derivative evaluations, provided that the nonlinear program is formulated with a linear objective and that the schedule for decreasing the barrier parameter is related in a certain way to the convergence criterion for each Newton process.
\end{abstract}

\section{Introduction}

We consider the nonlinear programming problem

$$
\min f(x) \quad \text { subject to } \quad c(x) \geq 0,
$$

where $f: \mathrm{R}^{n} \rightarrow \mathrm{R}$ and $c: \mathrm{R}^{n} \rightarrow \mathrm{R}^{m}$ are smooth (twice Lipschitz continuously differentiable) functions. The logarithmic barrier function for (1) is

$$
P(x ; \mu)=f(x)-\mu \sum_{i=1}^{m} \log c_{i}(x) .
$$

We denote by $x(\mu)$ a minimizer of $P(. ; \mu)$ for $\mu>0$ and assume that $x(\mu)$ exists for all sufficiently small $\mu$. Methods based on (2) approximate $x(\mu)$ for a sequence of small, decreasing values of $\mu>0$. Under certain conditions (see Fiacco and McCormick [10]), we have $\lim _{\mu \downarrow 0} x(\mu)=x^{*}$, where $x^{*}$ is a local minimizer of (1).

The Newton/log-barrier method proceeds by fixing $\mu$ at a certain value and applying Newton's method to the unconstrained problem

$$
\min _{x} P(x ; \mu)
$$

Stephen J. Wright: Mathematics and Computer Science Division, Argonne National Laboratory, 9700 South Cass Avenue, Argonne, Illinois 60439; rright@mcs.anl.gov

Mathematics Subject Classification (1991): ??, ??, ?? 
stopping the Newton iterations when some tolerance is satisfied, giving an approximation to $x(\mu)$. (Typically, the size of the Newton step or of the gradient $P_{x}(x ; \mu)$ is required to fall below a certain threshold that depends on $\mu$.) The barrier parameter $\mu$ is then decreased (to $\mu_{+}$, say), and Newton's method is applied to $P\left(\cdot ; \mu_{+}\right)$. In some algorithms, a different type of step such as a primal-dual or extrapolation step is used at the first iteration after reduction to $\mu_{+}$.

When the final approximation to $x(\mu)$ is used as a starting point for Newton's method applied to $P\left(\cdot ; \mu_{+}\right)$, it is well known that the first Newton step for each value of $\mu$ usually is a poor search direction, and a step length $\alpha$ considerably smaller than 1 usually is needed to remain feasible at this iteration (see Conn, Gould, and Toint [5], M. Wright [26], and S. Wright and Jarre [29]). Often, however, subsequent iterations of Newton's method converge rapidly to $x(\mu)$. Although the Hessian $P_{x x}(x ; \mu)$ is positive definite near $x=x(\mu)$ (see the proof of [10, Theorem 12]), the observed rate of convergence of Newton's method is better than we might expect from a naive application of the standard local convergence theory, which implies that the largest Euclidean ball within which quadratic convergence can be expected has radius $O\left(\mu^{2}\right)$. In fact, as we show in Section 4, Newton's method with unit steps converges from all points in a Euclidean neighborhood of radius $O\left(\mu^{\sigma}\right)$, for any $\sigma>1$ and all $\mu$ sufficiently small. In a sense, this radius is as large as we could expect for any minimization algorithm applied to $P(\cdot ; \mu)$, because Euclidean balls with radii proportional to smaller powers of $\mu$ ( $\mu^{1}$, for instance) may contain infeasible points for (1), which lie outside the domain of $P(\cdot ; \mu)$.

In Section 5, we discuss a line-search variant of Newton's method and specify typical conditions that are required of the line-search parameter. We show that when the iterates lie in neighborhoods of the type discussed in Section 4, the unit step length satisfies these conditions for all sufficiently small values of $\mu$.

In Section 6, we use earlier results of S. Wright and Jarre [29] to show that, when the objective function $f$ is linear and the line search and stopping criteria for the Newton iterations are defined in a certain reasonable way, then the first Newton step taken after each substantial reduction of $\mu$ produces an iterate that is within the quadratic convergence domain of Section 4. Compiling all our results, we specify a particular line-search Newton/log-barrier methodAlgorithm NLB - and show that for particular choices of the parameters, this algorithm converges at an overall superlinear rate.

In Section 2, we discuss related work on log-barrier and interior-point methods and put our results and Algorithm NLB into perspective. Section 3 outlines our assumptions and a basic lemma.

We use the following notation in the rest of the paper. For related positive quantities $\alpha$ and $\beta$, we say $\beta=O(\alpha)$ if there is a constant $M$ such that $\beta \leq M \alpha$ for all $\alpha$ sufficiently small. We say that $\beta=o(\alpha)$ if $\beta / \alpha \rightarrow 0$ as $\alpha \rightarrow 0, \beta=\Omega(\alpha)$ if $\alpha=O(\beta)$, and $\beta=\Theta(\alpha)$ if $\beta=O(\alpha)$ and $\alpha=O(\beta)$. It follows that the expression $\beta=O(1)$ means that $\beta \leq M$ for some constant $M$ and all values of $\beta$ in the domain of interest. 


\section{Motivation}

A good deal of literature exists on variants of the Newton/log-barrier method, starting with the book of Fiacco and McCormick [10]. Our focus in this paper is on variants of the method in which all the steps are line-search Newton steps for the primal barrier function $P(\cdot ; \mu)$, for various values of $\mu$. That is, no primal-dual or extrapolation steps are taken to enhance the overall convergence after each reduction of $\mu$. We are motivated partly by a desire to keep the algorithm simple, freeing it from devices that improve local convergence rate without having an intuitive motivation or relevance at iterates far from the solution. Another reason for our continued investigation of the primal log-barrier approach is that most of the effort in designing and implementing primal-dual algorithms (which have greater practical potential than primal methods) has focused on global convergence issues, and the merit functions that are used to ensure global convergence are usually modifications of the purely primal function (2). See, for example, the merit functions proposed by Forsgren and Gill [12], Byrd, Hribar, and Nocedal [3], Gay, Overton, and M. Wright [13], and Conn et al. [4].

The approach of using only Newton steps for $P(\cdot ; \mu)$ in the algorithm-in particular, of using the approximate minimizer of $P(\cdot ; \mu)$ for one value of $\mu$ as the starting point for Newton's method at the next value of $\mu$-is consistent with the approach specified in Fiacco and McCormick [10, p. 42] and also with approaches described and analyzed by other authors, notably, M. Wright [24, p. 389], [26, Section 2.2] and Conn et al. [4]. Nash and Sofer [22, Section 7] indicate that when default parameter settings were used, the algorithm implemented in the SUMT software (produced by Fiacco, McCormick, and their collaborators) is also consistent with the type of method that we analyze (with the exception that the Newton method can be replaced by quasi-Newton or steepest-descent in the local search.)

Methods based on the modern theory of self-concordant barrier functions are also consistent with the approach we analyze here, in that they take only linesearch Newton steps for the barrier function, for a monotonic sequence of barrier values. Nesterov and Nemirovskii [23] discuss this approach for various types of convex programming problems, considering both short-step methods (in which each modest reduction in $\mu$ is followed by a single unit-length step of Newton's method) and long-step methods (in which more substantial reductions in $\mu$ are made, and a number of line-search Newton steps taken for $P(\cdot ; \mu)$ for each value of $\mu$ ).

The literature on self-concordant barrier methods typically assumes a linear objective function (for example, [23, p. 57]), as we do also in Section 6 of this paper. A simple reformulation can be used to express any nonlinear program (1) in this form: we can introduce the artificial variable $\eta$ and the new constraint $\eta-f(x) \geq 0$ and replace the objective in (1) by $\eta$. In the self-concordant setting, the reason for assuming linear objective is that the second-order and higher derivatives of the barrier function for the optimization problem do not contain any contribution from the objective and are linear in the barrier parameter. As a result of the latter property, the self-concordant property for the barrier 
function becomes independent both of the barrier parameter and of the objective; it depends only on the feasible set and possibly its algebraic representation. Our reasons for requiring a linear objective in Section 6 are similar, in that by doing so we make the Newton step linear in $\mu^{-1}$ (a fact that can be verified easily from (2) by calculating the Newton step for $\mu^{-1} P(\cdot ; \mu)$ ) and consequently much easier to analyze. The analysis of the linear-objective case is the focus of S. Wright and Jarre [29].

We believe our results in Section 6 to be novel in that rapid local convergence of primal barrier algorithms that use only Newton search directions for $P(\cdot ; \mu)$ apparently has not been described before, either in the context of nonlinear programming or in convex programming applications of the type considered by Nesterov and Nemirovskii [23]. However, rapid convergence of methods for (1) based on the log-barrier function (2) that take other kinds of steps has been described in a number of papers. In the main, these papers address the poor performance of Newton's method at the step taken immediately after a reduction of the barrier parameter by using some other method to generate this step. Conn, Gould, and Toint [5] advocate taking a primal-dual step, obtained by applying Newton's method for nonlinear equations to the system (9), treating $x$ and $\lambda$ as independent variables. Gould [15] applies a similar strategy to the quadratic penalty function for equality-constrained optimization. The related approach is to take an approximate extrapolation step along the path of minimizers $\{x(\mu) \mid \mu>0\}$, so that the first step taken after reducing $\mu$ to $\mu_{k+1}$, say, is obtained by fitting a polynomial through the approximate minimizers $x_{k}, x_{k-1}, \ldots$ at the barrier parameter values $\mu_{k}, \mu_{k-1}, \ldots$ or by computing information about the tangent $\dot{x}(\mu)$ at $x_{k}$. Techniques of this class were suggested by Fiacco and McCormick [10]; see also Nash and Sofer [21], Benchakroun, Dussault, and Mansouri [1], and Dussault [9]. The papers [1] and [9] show that "superlinear" reductions in $\mu$, and hence rapid convergence to $x^{*}$, can be attained. In fact, the method of Benchakroun, Dussault, and Mansouri [1] generates identical iterates to Algorithm NLB, for a version of this algorithm in which we make a particular choice of the initial step length along the first Newton step taken after each reduction of $\mu$. In a slightly different vein, Fiacco and McCormick [10] and Jittorntrum and Osborne [17], show that extrapolation can also be used to obtain successive estimates of $x^{*}$, but their techniques assume that the minimizations of $P(x ; \mu)$ are carried out accurately for each value of $\mu$.

A related class of methods, known as analytic center methods, uses Newton's method to find the successive minima of a certain logarithmic potential function that is parametrized by an upper bound on the optimal value of $f$ and possibly by a weighting term. The resulting function is similar (but not identical) to one that would be obtained by applying the reformulation discussed above in which the objective $f(x)$ is replaced by the linear objective $\eta$ and the constraint $\eta-f(x) \geq 0$. The convergence of Newton's method in this context has been examined by Nesterov and Nemirovskii [23], Jarre [16], and Den Hertog, Roos, and Terlaky [6], and others. The cited papers consider a special class of problems in which the objective function and constraints are convex and satisfy a certain scaled Lipschitz or self-concordancy condition, whereas our analysis applies lo- 
cally to general functions under smoothness assumptions that are standard in the local analysis of nonlinear programming methods. To the best of our knowledge, superlinear convergence has not been considered for analytic center methods.

In Section 7, we discuss all these methods and results further and relate them to the results of this paper.

\section{Assumptions}

In this section, we specify the optimality conditions for the nonlinear program (1), outline our assumptions on its solution $x^{*}$, and introduce some notation.

The Lagrangian function for (1) is

$$
\mathcal{L}(x, \lambda)=f(x)-\lambda^{T} c(x),
$$

where $\lambda$ is the vector of Lagrange multipliers. The solution $x^{*}$ of (1) satisfies the first-order conditions for optimality, which are that there exists a Lagrange multiplier vector $\lambda^{*}$ such that

$$
c\left(x^{*}\right) \geq 0, \quad \lambda^{*} \geq 0, \quad\left(\lambda^{*}\right)^{T} c\left(x^{*}\right)=0, \quad \nabla f\left(x^{*}\right)=\sum_{i=1}^{m} \lambda_{i}^{*} \nabla c_{i}\left(x^{*}\right) .
$$

The active constraints are the components of $c$ for which $c_{i}\left(x^{*}\right)=0$. Without loss of generality we assume these to be the first $q$ components of $c$. We also assume that the solution is nondegenerate, that is,

$$
\left[\nabla c_{1}\left(x^{*}\right)|\cdots| \nabla c_{q}\left(x^{*}\right)\right] \text { has rank } q .
$$

(Note that nondegeneracy implies uniqueness of $\lambda^{*}$.) We also assume strict complementarity, that is,

$$
\lambda_{i}^{*}+c_{i}\left(x^{*}\right)>0, \quad i=1,2, \ldots, m .
$$

Finally, we assume that second-order sufficient conditions for optimality are satisfied at $\left(x^{*}, \lambda^{*}\right)$, that is,

$$
y^{T} \mathcal{L}_{x x}\left(x^{*}, \lambda^{*}\right) y>0 \text { for all } y \neq 0 \text { with } \nabla c_{i}\left(x^{*}\right)^{T} y=0 \text { for all } i=1,2, \ldots, q .
$$

It is easy to see that $\left(x^{*}, \lambda^{*}\right)$ is a root of the function $F(x, \lambda)$ defined by

$$
F(x, \lambda)=\left[\begin{array}{c}
\mathcal{L}_{x}(x, \lambda) \\
\Lambda c(x)
\end{array}\right]=\left[\begin{array}{c}
g-A(x) \lambda \\
\Lambda c(x)
\end{array}\right]
$$

where

$$
\Lambda=\operatorname{diag}\left(\lambda_{1}, \lambda_{2}, \cdots, \lambda_{m}\right)
$$

The Jacobian of $F$ is

$$
\nabla F(x, \lambda)=\left[\begin{array}{cc}
\mathcal{L}_{x x}(x, \lambda) & -A(x) \\
\Lambda A(x)^{T} & C(x)
\end{array}\right],
$$


where $C(x)=\operatorname{diag}\left(c_{1}(x), c_{2}(x), \cdots, c_{m}(x)\right)$. When the nondegeneracy, strict complementarity, and second-order sufficient conditions hold, $\nabla F\left(x^{*}, \lambda^{*}\right)$ is nonsingular. It follows from the assumed smoothness of $f$ and $c$ that the Jacobian $\nabla F(x, \lambda)$ is nonsingular for all $(x, \lambda)$ close to $\left(x^{*}, \lambda^{*}\right)$ (see Fiacco and McCormick [10, Theorem 14]).

Given any strictly feasible point $x$ and any positive value of the barrier parameter $\mu$ in (2), we define a vector of Lagrange multiplier estimates $\lambda(x, \mu)$ by

$$
\lambda(x, \mu)=\mu C(x)^{-1} e=\left[\frac{\mu}{c_{1}(x)}, \ldots, \frac{\mu}{c_{m}(x)}\right]^{T} .
$$

If $x$ is the exact minimizer $x(\mu)$ of $P(\cdot ; \mu)$, we define

$$
\lambda(\mu) \triangleq \lambda(x(\mu), \mu) .
$$

For future reference, we note that the derivatives of the barrier function (2) are

$$
\begin{aligned}
P_{x}(x ; \mu) & =\nabla f(x)-\sum_{i=1}^{m} \frac{\mu}{c_{i}(x)} \nabla c_{i}(x) \\
P_{x x}(x ; \mu) & =\nabla^{2} f(x)+\mu \sum_{i=1}^{m}\left[\frac{1}{c_{i}^{2}(x)} \nabla c_{i}(x) \nabla c_{i}(x)^{T}-\frac{1}{c_{i}(x)} \nabla^{2} c_{i}(x)\right] .
\end{aligned}
$$

\section{Convergence of Newton's Method with Unit Steps to the Log-Barrier Minimizer}

We now analyze the local convergence properties of Newton's method with unit steps to the minimizer $x(\mu)$ of the barrier function $P(x ; \mu)$, for a fixed value of $\mu$. From the current iterate $w$, the Newton step $s$ is

$$
s=-P_{x x}(w ; \mu)^{-1} P_{x}(w ; \mu),
$$

and the next iterate is $w_{+}=w+s$. In this section, we identify a Euclidean ball around the minmizer of $P(\cdot ; \mu)$ such that when the unit-step Newton method is started from any point in this ball, it converges rapidly to the minimizer.

It is well known that under the second-order assumptions discussed above, the barrier function $P(x ; \mu)$ has a minimizer $x(\mu)$ at which the Hessian is positive definite, though ill conditioned. Moreover, since the objective $f(\cdot)$ and constraint functions $c_{i}(\cdot), i=1,2, \ldots, m$ are twice Lipschitz continuously differentiable, then $P(x ; \mu)$ is also twice Lipschitz continuously differentiable near $x(\mu)$. Hence, quadratic convergence follows from a standard result (see, for example, Theorem 5.2.1 of Dennis and Schnabel [8]). If $w_{1}, w_{2}, w_{3}, \ldots$ are the Newton iterates, the standard theory yields the estimate

$$
\left\|w_{t+1}-x(\mu)\right\| \leq L_{1}(\mu) L_{2}(\mu)\left\|w_{t}-x(\mu)\right\|^{2},
$$


where $L_{1}(\mu)$ is a Lipschitz constant for $P_{x x}(x ; \mu)$ in the vicinity of $x(\mu)$ and $L_{2}(\mu)$ is a bound on $\left\|P_{x x}(x ; \mu)^{-1}\right\|$ near $x(\mu)$. In fact, we have that

$$
L_{1}(\mu)=O\left(\mu^{-2}\right), \quad L_{2}(\mu)=O(1) .
$$

(The estimate of $L_{2}$ is a consequence of the analysis in Murray [19] and the second-order sufficient conditions (8)). Therefore, (15) reduces to

$$
\left\|w_{t+1}-x(\mu)\right\|=O\left(\mu^{-2}\right)\left\|w_{t}-x(\mu)\right\|^{2} .
$$

This expression does not even imply convergence of the iteration sequence unless $w_{1}$ is in a very small neighborhood of the solution, specifically,

$$
\left\|w_{1}-x(\mu)\right\|=O\left(\mu^{2}\right) .
$$

S. Wright and Jarre [29] investigated the use of a reformulation of (1) in which the objective function is linear. They show that if the final approximation to $x\left(\mu_{-}\right)$obtained at the previous value $\mu_{-}$of the barrier parameter is reasonably accurate, then the Newton step for $P(\cdot ; \mu)$ from this point for the new value of $\mu$ passes quite close to the new minimizer $x(\mu)$. Even in this case, however, the resulting point will not generally lie in the neighborhood (17), except possibly when a stringent stopping criterion of the form $\left\|P_{x}(x ; \mu)\right\|=O(\mu)$ is used at the previous value of $\mu$.

In this section, we show that the expressions (15) and (17) are unduly pessimistic and, in fact, that there exists a constant $\bar{\mu}_{0}>0$ such that quadratic convergence of Newton's method with unit steps can be obtained from any point $w$ that satisfies

$$
\|w-x(\mu)\| \leq C_{0} \mu^{\sigma}, \quad \text { for all } \mu \in\left(0, \bar{\mu}_{0}\right],
$$

where $C_{0}$ and $\sigma$ are certain constants satisfying $C_{0}>0$ and $\sigma>1$. That is, the domain of quadratic convergence for $P(\cdot ; \mu)$ shrinks as $\mu \downarrow 0$, but the rate of shrinkage is not especially severe. Key to our analysis is a partitioning of the space $\mathbf{R}^{n}$ into the range space of the active constraint Jacobian and its complement. A decomposition of this type has been used previously (for example, by M. Wright [25-27]) to analyze the properties of the gradient and Hessian of $P(\cdot ; \mu)$; we mention some specific connections below.

We assume a priori that $w$ lies in the neighborhood

$$
\|w-x(\mu)\| \leq C \mu^{\sigma},
$$

where $C>0$ and $\sigma>1$ are given constants, and that

$$
\mu \in(0, \bar{\mu}],
$$

for some $\bar{\mu}>0$. We show later in the section how $C_{0}$ and $\bar{\mu}_{0}$ depend on $C$ and $\bar{\mu}$. 
The analysis is based on the following consequence of Taylor's theorem. If $w$ is the current iterate and $s$ is the Newton step, as in (14), we have that

$$
\begin{aligned}
P_{x}(w+s ; \mu) & =P_{x}(w ; \mu)+P_{x x}(w ; \mu) s+\int_{0}^{1}\left[P_{x x}(w+\tau s ; \mu)-P_{x x}(w ; \mu)\right] s d \tau \\
& =\int_{0}^{1}\left[P_{x x}(w+\tau s ; \mu)-P_{x x}(w ; \mu)\right] s d \tau
\end{aligned}
$$

By analyzing the integral term, we obtain estimates for the projection of $P_{x}(w+$ $s ; \mu)$ into two complementary subspaces induced by the Jacobian of the active constraint matrix. We then investigate the two-sided projection of $P_{x x}(w+s ; \mu)$ in these same subspaces to obtain a bound on the size of the next Newton step-the one taken from the point $w+s$.

We assume without loss of generality that $\bar{\mu}$ is small enough that the neighborhood (19) excludes local minimizers of $P(\cdot ; \mu)$ other than $x(\mu)$. Since $\sigma>1$ in (19), it follows that the ratio $\|w-x(\mu)\| / \mu$ approaches zero as $\mu \downarrow 0$.

It follows immediately from the existence and boundedness of $(\dot{x}(\mu), \dot{\lambda}(\mu))$ for all sufficiently small $\mu$ (see Fiacco and McCormick [10, Theorem 15]) that

$$
\left\|\left[\begin{array}{c}
x(\mu)-x^{*} \\
\lambda(\mu)-\lambda^{*}
\end{array}\right]\right\|=O(\mu)
$$

Hence, by using (11), the strict complementarity assumption, and (19), we have for all active indices $i=1,2, \ldots, q$ that

$$
\begin{aligned}
& c_{i}(w)=c_{i}(x(\mu))+O(\|w-x(\mu)\|) \\
& =\frac{\mu}{\lambda_{i}(\mu)}+O\left(\mu^{\sigma}\right)=\frac{\mu}{\lambda_{i}^{*}}+O\left(\mu^{\min (2, \sigma)}\right)=\Theta(\mu) . \quad i=1,2, \ldots, q,
\end{aligned}
$$

for all $\mu$ sufficiently small.

Using this observation, we examine the relevant properties of $P_{x x}(w ; \mu)$. The nature of the ill conditioning in this matrix at minimizing points $w=x(\mu)$ was examined by Lootsma [18] and Murray [19]. M. Wright [25] further examined the eigenstructure of this matrix in a strictly feasible neighborhood of the solution $x^{*}$, for small $\mu$, and essentially showed in [25, Lemmas 3.1, 3.2, Theorem 3.1] that this matrix has $q$ eigenvalues of size $\Theta\left(\mu^{-1}\right)$ and $n-q$ eigenvalues of size $O(1)$. Drawing on Murray [19], M. Wright [25, Theorem 3.3] shows further that the $n-q$ small eigenvalues are of size $\Theta\left(\mu^{-1}\right)$ at $x=x(\mu)$. We now show that similar eigenvalue estimates hold on the neighborhood (19), and we derive explicit expressions for the Hessian and its inverse based on the two-sided projection induced by the active constraint Jacobian $\left[\nabla c_{i}(w)\right]_{i=1}^{q}$.

From (13b), we obtain by partitioning the sums into active and inactive indices that

$$
\begin{gathered}
P_{x x}(w ; \mu)=\sum_{i=1}^{q} \frac{\mu}{c_{i}^{2}(w)} \nabla c_{i}(w) \nabla c_{i}(w)^{T}+\left\{\nabla^{2} f(w)-\sum_{i=1}^{q} \frac{\mu}{c_{i}(w)} \nabla^{2} c_{i}(w)\right\} \\
-\sum_{i=q+1}^{m}\left\{\frac{\mu}{c_{i}^{2}(w)} \nabla c_{i}(w) \nabla c_{i}(w)^{T}-\frac{\mu}{c_{i}(w)} \nabla^{2} c_{i}(w)\right\} .
\end{gathered}
$$


We deal with the three terms on the right-hand side in turn. Because of (23), we have

$$
\frac{\mu}{c_{i}^{2}(w)}=\Theta\left(\mu^{-1}\right), \quad i=1,2, \ldots, q,
$$

so by the nondegeneracy assumption, the first sum in (24) is a rank- $q$ matrix, whose $q$ nonzero eigenvalues are all positive with size $\Theta\left(\mu^{-1}\right)$. (This claim follows from [25, Lemma 3.1].) Using a standard factorization, we can write

$$
\sum_{i=1}^{q} \frac{\mu}{c_{i}^{2}(w)} \nabla c_{i}(w) \nabla c_{i}(w)^{T}=\hat{U}(w) \hat{D}(w) \hat{U}(w)^{T},
$$

where $\hat{D}(w)$ is a $q \times q$ diagonal matrix whose diagonal elements all have size $\Theta\left(\mu^{-1}\right)$ and $\hat{U}(w)$ is an $n \times q$ orthonormal matrix whose columns span the range space of $\left[\nabla c_{i}(w)\right]_{i=1}^{q}$.

Since $\|w-x(\mu)\| / \mu=o(1)$, it follows that $\mu / c_{i}(w) \approx \lambda_{i}^{*}$, and the second term in (24) is a small perturbation of the Lagrangian Hessian $\mathcal{L}_{x x}\left(x^{*}, \lambda^{*}\right)$, which by our second-order assumption (8) is positive definite on the null space of $\left[\nabla c_{i}\left(x^{*}\right)\right]_{i=1}^{q}$. Hence, if we define $\tilde{U}(w)$ to be an $n \times(n-q)$ orthonormal matrix that spans the nearby null space of $\left[\nabla c_{i}(w)\right]_{i=1}^{q}$ (so that $[\hat{U}(w) \mid \tilde{U}(w)]$ is orthogonal), straightforward arguments show that the $(n-q) \times(n-q)$ matrix $G_{22}(w)$ defined by

$$
\begin{aligned}
\tilde{G}_{22}(w) & \triangleq \tilde{U}(w)^{T}\left\{\nabla^{2} f(w)-\sum_{i=1}^{q} \frac{\mu}{c_{i}(w)} \nabla^{2} c_{i}(w)\right\} \tilde{U}(w) \\
& =\tilde{U}(w)^{T} \mathcal{L}_{x x}\left(x^{*}, \lambda^{*}\right) \tilde{U}(w)+o(1)
\end{aligned}
$$

is positive definite, with all eigenvalues of size $\Theta(1)$.

Since $c_{i}(w)=\Omega(1)$ for $i=q+1, \ldots, m$ and all $\mu$ sufficiently small, the third term in $(24)$ is $O(\mu)$. We see therefore that $P_{x x}(w ; \mu)$ is an $O(1)$ perturbation of the matrix in $(25)$, so that $P_{x x}(w ; \mu)$ has $q$ eigenvalues of size $\Theta\left(\mu^{-1}\right)$ and $n-q$ eigenvalues of size $O(1)$. We now go on to show with the aid of a technical result that in fact the small eigenvalues are all positive with size $\Theta(1)$, when $w$ lies in the neighborhood (19).

By combining all these observations about the three terms in (24), we find that

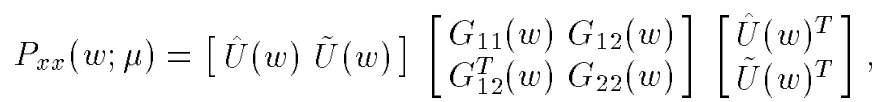

where

$$
\begin{aligned}
& G_{11}(w) \triangleq \hat{U}(w)^{T} P_{x x}(w ; \mu) \hat{U}(w)=\hat{D}(w)+O(1), \\
& G_{22}(w) \triangleq \tilde{U}(w)^{T} P_{x x}(w ; \mu) \tilde{U}(w)=\tilde{G}_{22}(w)+O(\mu), \\
& G_{12}(w) \triangleq \hat{U}(w)^{T} P_{x x}(w ; \mu) \tilde{U}(w)=O(1),
\end{aligned}
$$

where all the matrices in these expressions are defined in (25), (26), and the discussion above. 
It follows immediately from (28) that

$$
\left\|P_{x x}(w ; \mu)\right\|=O\left(\mu^{-1}\right) .
$$

To uncover the properties of the inverse $P_{x x}(w ; \mu)^{-1}$, we use the following technical result about the inverse of a block $2 \times 2$ matrix.

Lemma 1. Let $G$ be a symmetric matrix partitioned as

$$
G=\left[\begin{array}{ll}
G_{11} & G_{12} \\
G_{12}^{T} & G_{22}
\end{array}\right]
$$

where $G_{11}$ and $G_{22}$ are square. Suppose that $G_{11}$ and $G_{22}-G_{12}^{T} G_{11}^{-1} G_{12}$ are nonsingular. Then $G$ is nonsingular and $G^{-1}$ has the form

$$
G^{-1}=\left[\begin{array}{ll}
H_{11} & H_{12} \\
H_{12}^{T} & H_{22}
\end{array}\right]
$$

where

$$
\begin{aligned}
& H_{11}=G_{11}^{-1}+G_{11}^{-1} G_{12}\left(G_{22}-G_{12}^{T} G_{11}^{-1} G_{12}\right)^{-1} G_{12}^{T} G_{11}^{-1} \\
& H_{12}=-G_{11}^{-1} G_{12}\left(G_{22}-G_{12}^{T} G_{11}^{-1} G_{12}\right)^{-1} \\
& H_{22}=\left(G_{22}-G_{12}^{T} G_{11}^{-1} G_{12}\right)^{-1}
\end{aligned}
$$

In our case-the $2 \times 2$ block matrix in (28)-we have from the fact that the diagonals of $\hat{D}(w)$ have size $\Theta\left(\mu^{-1}\right)$ that

$$
G_{11}(w)^{-1}=\hat{D}(w)^{-1}(I+O(\mu))^{-1}=\hat{D}(w)^{-1}+O\left(\mu^{2}\right)=O(\mu) .
$$

By using this estimate, we further obtain from (28) and (26) that

$$
\begin{aligned}
& {\left[G_{22}(w)-G_{12}(w)^{T} G_{11}(w)^{-1} G_{12}(w)\right]^{-1}} \\
& =\left[\tilde{G}_{22}(w)+O(\mu)\right]^{-1}=\tilde{G}_{22}(w)^{-1}+O(\mu)=O(1)
\end{aligned}
$$

and that all the $m-q$ eigenvalues of this matrix are positive with size $\Theta(1)$. Hence, from (27) and (30), we obtain

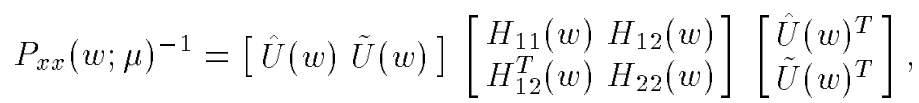

where

$$
H_{11}(w)=O(\mu), \quad H_{12}(w)=O(\mu), \quad H_{22}(w)=O(1)
$$

Note in particular that for sufficiently small $\mu$, the largest $n-q$ eigenvalues of the matrix $H(w)$ are $O(\mu)$ of the $n-q$ eigenvalues of $\tilde{G}_{22}(w)^{-1}$, which by (26) are all of size $\Theta(1)$ and positive. Hence the smallest $n-q$ eigenvalues of $G(w)$ are all of size $\Theta(1)$ and positive.

We summarize the analysis of the paragraphs above as a lemma. 
Lemma 2. Let the constants $C>0$ and $\sigma>1$ in (19) be given. Then there is a $\bar{\mu}>0$ such that for all $w$ and $\mu$ satisfying (19) and (20), the Hessian $P_{x x}(w ; \mu)$ is positive definite, and this matrix and its inverse have the structure shown in (27), (28) and (31), (32), respectively.

We now examine the properties of $P_{x}(w ; \mu)$. From (19) and (23) we have (by decreasing $\bar{\mu}$ if necessary) that

$$
\|w-x(\mu)\| / c_{i}(w)=O\left(\mu^{\sigma-1}\right) \ll 1, \quad i=1,2, \ldots, q,
$$

for all $\mu$ satisfying (20). We can use this expression to estimate the difference between the reciprocals $c_{i}^{-1}(w)$ and $c_{i}^{-1}(x(\mu))$. We have

$$
\begin{aligned}
c_{i}^{-1}(x(\mu)) & =\left[c_{i}(w)+O(\|w-x(\mu)\|)\right]^{-1} \\
& =c_{i}^{-1}(w)\left[1+O\left(c_{i}(w)^{-1}\|w-x(\mu)\|\right)\right]^{-1} \\
& =c_{i}^{-1}(w)+c_{i}^{-2}(w) O(\|w-x(\mu)\|) .
\end{aligned}
$$

From (2), noting that $P_{x}(x(\mu) ; \mu)=0$ and partitioning active and inactive indices, we have that

$$
\begin{aligned}
P_{x}(w ; \mu)= & P_{x}(w ; \mu)-P_{x}(x(\mu) ; \mu) \\
= & \sum_{i=1}^{q}\left[\frac{\mu}{c_{i}(w)} \nabla c_{i}(w)-\frac{\mu}{c_{i}(x(\mu))} \nabla c_{i}(x(\mu))\right] \\
& +[\nabla f(w)-\nabla f(x(\mu))] \\
& \quad+\sum_{i=q+1}^{m}\left[\frac{\mu}{c_{i}(w)} \nabla c_{i}(w)-\frac{\mu}{c_{i}(x(\mu))} \nabla c_{i}(x(\mu))\right] .
\end{aligned}
$$

For the second term on the right-hand side of (35), we have by smoothness of $f$ that $\nabla f(w)-\nabla f(x(\mu))=O(\|w-x(\mu)\|)$. In the third term, we have for each index $i=q+1, \ldots, m$ that $c_{i}(w)=\Omega(1)$ and $c_{i}(x(\mu))=\Omega(1)$. Hence, by smoothness of $\nabla c_{i}$, this term has size $O(\mu\|w-x(\mu)\|)$.

In the first term, we have for each active index $i$, using the smoothness of $\nabla c_{i}$ together with (23), (33), and (34), that

$$
\begin{aligned}
& \frac{\mu}{c_{i}(x(\mu))} \nabla c_{i}(x(\mu)) \\
& =\left[\frac{\mu}{c_{i}(w)}+\frac{\mu}{c_{i}^{2}(w)} O(\|w-x(\mu)\|)\right]\left[\nabla c_{i}(w)+O(\|w-x(\mu)\|)\right] \\
& =\frac{\mu}{c_{i}(w)} \nabla c_{i}(w)+O\left(\mu^{-1}\|w-x(\mu)\|\right) \nabla c_{i}(w)+O(\|w-x(\mu)\|) .
\end{aligned}
$$

By collecting these observations and substituting into (35), we obtain

$$
P_{x}(w ; \mu)=\sum_{i=1}^{q} O\left(\mu^{-1}\|w-x(\mu)\|\right) \nabla c_{i}(w)+O(\|w-x(\mu)\|) .
$$


From the definitions of $\hat{U}(w)$ and $\tilde{U}(w)$, it follows immediately that

$$
\begin{aligned}
& \hat{U}(w)^{T} P_{x}(w ; \mu)=O\left(\mu^{-1}\|w-x(\mu)\|\right), \\
& \tilde{U}(w)^{T} P_{x}(w ; \mu)=O(\|w-x(\mu)\|) .
\end{aligned}
$$

Meanwhile from (32), we have for the Newton step from $w$ that

$$
\begin{aligned}
& s=-P_{x x}(w ; \mu)^{-1} P_{x}(w ; \mu) \\
& =[\hat{U}(w) \tilde{U}(w)]\left[\begin{array}{ll}
H_{11}(w) & H_{12}(w) \\
H_{12}^{T}(w) & H_{22}(w)
\end{array}\right]\left[\begin{array}{l}
\hat{U}(w)^{T} P_{x}(w ; \mu) \\
\tilde{U}(w)^{T} P_{x}(w ; \mu)
\end{array}\right] \\
& =[\hat{U}(w) \tilde{U}(w)]\left[\begin{array}{ll}
O(\mu) & O(\mu) \\
O(\mu) & O(1)
\end{array}\right]\left[\begin{array}{l}
\hat{U}(w)^{T} P_{x}(w ; \mu) \\
\tilde{U}(w)^{T} P_{x}(w ; \mu)
\end{array}\right] .
\end{aligned}
$$

Hence, by combining with (38), we find that we can choose $\bar{\mu}$ in (20) such that for all $w$ and $\mu$ satisfying (19), we have

$$
\begin{aligned}
\|s\| & \leq O\left(\mu\left\|\hat{U}(w)^{T} P_{x}(w ; \mu)\right\|+\left\|\tilde{U}(w)^{T} P_{x}(w ; \mu)\right\|\right) \\
& \leq C_{3}\left[\mu\left\|\hat{U}(w)^{T} P_{x}(w ; \mu)\right\|+\left\|\tilde{U}(w)^{T} P_{x}(w ; \mu)\right\|\right] \\
& \leq C_{1}\|w-x(\mu)\|=O\left(\mu^{\sigma}\right),
\end{aligned}
$$

for some positive numbers $C_{1}$ and $C_{3}$ independent of $\mu$.

Note that the naive estimate of $\|s\|$ obtained by ignoring the structure of $P_{x}(w ; \mu)$ and $P_{x x}(w ; \mu)$ would be simply $\|s\| \leq\left\|P_{x x}(w ; \mu)^{-1}|||| P_{x}(w ; \mu)\right\|=$ $O\left(\mu^{\sigma-1}\right)$, which is too pessimistic for our purposes.

We now examine the integrand in (21), partitioning it into the subspaces defined by the active constraint gradients at the next Newton iterate $w+s$. We start by partitioning the integrand as follows:

$$
\left[P_{x x}(w+\tau s ; \mu)-P_{x x}(w ; \mu)\right] s=r_{1}+r_{2}+r_{3}+r_{4},
$$

where $\tau \in[0,1]$ and

$$
\begin{aligned}
r_{1}= & \sum_{i=1}^{q}\left\{\frac{\mu}{c_{i}^{2}(w+\tau s)} \nabla c_{i}(w+\tau s) \nabla c_{i}(w+\tau s)^{T} s-\frac{\mu}{c_{i}^{2}(w)} \nabla c_{i}(w) \nabla c_{i}(w)^{T} s\right\} \\
r_{2}= & -\sum_{i=1}^{q}\left\{\frac{\mu}{c_{i}(w+\tau s)} \nabla^{2} c_{i}(w+\tau s) s-\frac{\mu}{c_{i}(w)} \nabla^{2} c_{i}(w) s\right\} \\
r_{3}= & {\left[\nabla^{2} f(w+\tau s)-\nabla^{2} f(w)\right] s, } \\
r_{4}= & \sum_{i=q+1}^{m}\left\{\frac{\mu}{c_{i}^{2}(w+\tau s)} \nabla c_{i}(w+\tau s) \nabla c_{i}(w+\tau s)^{T} s-\frac{\mu}{c_{i}^{2}(w)} \nabla c_{i}(w) \nabla c_{i}(w)^{T} s\right. \\
& \left.-\frac{\mu}{c_{i}(w+\tau s)} \nabla^{2} c_{i}(w+\tau s) s+\frac{\mu}{c_{i}(w)} \nabla^{2} c_{i}(w) s\right\} .
\end{aligned}
$$


To estimate the first term $r_{1}$, we note from (39b) that $\mu^{-1}\|s\|=O\left(\mu^{\sigma-1}\right) \ll 1$. Hence, as in (34), we have

$$
\begin{aligned}
\frac{1}{c_{i}(w+\tau s)} & =\frac{1}{c_{i}(w)}+\frac{1}{c_{i}^{2}(w)} O(\|\tau s\|)=\frac{1}{c_{i}(w)}+O\left(\mu^{-2}\|s\|\right), \quad i=1,2, \ldots \\
\frac{1}{c_{i}^{2}(w+\tau s)} & =\frac{1}{c_{i}^{2}(w)}\left[1+\frac{1}{c_{i}(w)} O(\|\tau s\|)\right]^{-2} \\
& =\frac{1}{c_{i}^{2}(w)}+O\left(\mu^{-3}\|s\|\right), \quad i=1,2, \ldots, q .
\end{aligned}
$$

By smoothness of $\nabla c_{i}$, we obtain

$$
\begin{aligned}
& \frac{\mu}{c_{i}^{2}(w+\tau s)} \nabla c_{i}(w+\tau s) \nabla c_{i}(w+\tau s)^{T} s \\
& =\left[\frac{\mu}{c_{i}^{2}(w)}+O\left(\mu^{-2}\|s\|\right)\right]\left[\nabla c_{i}(w)+O(\|s\|)\right]\left[\nabla c_{i}(w)^{T} s+O\left(\|s\|^{2}\right)\right] \\
& =\frac{\mu}{c_{i}^{2}(w)} \nabla c_{i}(w) \nabla c_{i}(w)^{T} s+O\left(\mu^{-2}\|s \mid\| \hat{U}(w)^{T} s \|\right) \nabla c_{i}(w)+O\left(\mu^{-1}|| s \|^{2}\right),
\end{aligned}
$$

where we have used $\left|\nabla c_{i}(w)^{T} s\right|=O\left(\| \hat{U}(w)^{T} s||\right)$, which follows from the definition of $\hat{U}(w)$ in (25). We have also used the fact that $\mu^{-1}|| s \| \ll 1$ (see (39b)) to absorb higher-order terms. By substituting into the definition of $r_{1}$, we obtain

$$
\begin{aligned}
r_{1} & =\sum_{i=1}^{q} O\left(\mu^{-2}\|s\|\left\|\hat{U}(w)^{T} s\right\|\right) \nabla c_{i}(w)+O\left(\mu^{-1}\|s\|^{2}\right) \\
& =\sum_{i=1}^{q} O\left(\mu^{-2}\|s\|^{2}\right) \nabla c_{i}(w+s)+O\left(\mu^{-1}\|s\|^{2}\right),
\end{aligned}
$$

where we have used $\left\|\hat{U}(w)^{T} s\right\| \leq\|s\|$, noting that the change of argument from $w$ to $w+s$ in the first term causes a perturbation that can be absorbed in the second term. (A minor variant of this analysis shows that the Lipschitz constant for $P_{x x}(\cdot ; \mu)$ is $O\left(\mu^{-2}\right)$, as claimed in (16).)

For the second term $r_{2}$ in (40), we have from (41a) that

$$
\begin{aligned}
\frac{\mu}{c_{i}(w+\tau s)} \nabla^{2} c_{i}(w+\tau s) s & =\left[\frac{\mu}{c_{i}(w)}+O\left(\mu^{-1}\|s\|\right)\right]\left[\nabla^{2} c_{i}(w) s+O\left(\|s\|^{2}\right)\right] \\
& =\frac{\mu}{c_{i}(w)} \nabla^{2} c_{i}(w) s+O\left(\mu^{-1}\|s\|^{2}\right) .
\end{aligned}
$$

The remaining terms $r_{3}$ and $r_{4}$ are less significant. By Lipschitz continuity of $\nabla f$, we have $r_{3}=O\left(\|s\|^{2}\right)$. In $r_{4}$, the denominators all have size $\Omega(1)$, so it is easy to show that $r_{4}=O\left(\mu\|s\|^{2}\right)$. 
By collecting all these estimates into (40), performing the integration, and substituting into (21), we obtain

$$
\begin{aligned}
P_{x}(w+s ; \mu) & =\sum_{i=1}^{q} O\left(\mu^{-2}\|s\|\left\|\hat{U}(w)^{T} s\right\|\right) \nabla c_{i}(w)+O\left(\mu^{-1}\|s\|^{2}\right) \\
& =\sum_{i=1}^{q} O\left(\mu^{-2}\|s\|^{2}\right) \nabla c_{i}(w+s)+O\left(\mu^{-1}\|s\|^{2}\right) .
\end{aligned}
$$

Hence, after a possible adjustment in $\bar{\mu}$ in (20), we have from (44b) that there is a positive number $C_{2}$ independent of $\mu$ (assumed without loss of generality to satisfy $C_{2} \geq 1$ ) such that

$$
\begin{aligned}
& \hat{U}(w+s)^{T} P_{x}(w+s ; \mu) \leq C_{2} \mu^{-2}\|s\|^{2}, \\
& \tilde{U}(w+s)^{T} P_{x}(w+s ; \mu) \leq C_{2} \mu^{-1}\|s\|^{2},
\end{aligned}
$$

for any orthonormal matrix $\hat{U}(w+s)$ that spans the column space of $\left[\nabla c_{i}(x+\right.$ $s)]_{i=1}^{q}$ and for any orthonormal matrix $\tilde{U}(w+s)$ such that $[\hat{U}(w+s) \mid \tilde{U}(w+s)]$ is orthogonal.

We summarize the observations above in the following result.

Lemma 3. Let the constants $C>0$ and $\sigma>1$ in (19) be given. Then there exist $a \bar{\mu}>0$ and constants $C_{1}, C_{2}, C_{3}$ such that for all $w$ and $\mu$ satisfying (19) and (20), the relationships (39a), (39b), and (45) are satisfied, where $s$ is the Newton step (14).

An important consequence of this lemma is that if the next Newton iterate $w+s$ also lies in the neighborhood (19), then we have from (39a) and (45) that the Newton step $s_{+}$calculated from $w+s$ satisfies

$$
\left\|s_{+}\right\| \leq 2 C_{2} C_{3} \mu^{-1}\|s\|^{2} .
$$

We now use all these estimates to show that if we choose the starting point $w_{1}$ for the Newton iteration in a slightly more restrictive neighborhood than (19), then all Newton iterates will remain inside the full neighborhood (19), and quadratic convergence of the Newton sequence to $x(\mu)$ will be observed. We state the result formally as a theorem.

Theorem 1. Let the constants $C>0$ and $\sigma>1$ be given, and let $C_{1}, C_{2}, C_{3}$, and $\bar{\mu}$ be defined as above, in such a way that the relationships (39a), (39b), (45), and (46) hold for all $w$ satisfying (19). Let the constants $C_{0}>0$ and $\bar{\mu}_{0}$ be chosen in such a way that the following inequalities are satisfied:

$$
\left(1+2 C_{1}\right) C_{0} \leq C, \quad 2 C_{0} C_{1} C_{2} C_{3} \bar{\mu}_{0}^{\sigma-1} \leq 1 / 4 .
$$

Then if $\mu \in\left(0, \bar{\mu}_{0}\right]$ and $w_{1}$ is any point that satisfies

$$
\left\|w_{1}-x(\mu)\right\| \leq C_{0} \mu^{\sigma}
$$


then Newton's method with unit steps, applied to the function $P(\cdot ; \mu)$ and starting from $w_{1}$, generates a sequence of steps $\left\{s_{t}\right\}_{t=1,2,3, \ldots}$ and iterates $\left\{w_{t}\right\}_{t=1,2,3}$. such that

$$
\left\|w_{t+1}-x(\mu)\right\| \leq C_{4} \mu^{-1}|| w_{t}-x(\mu) \|^{2}, \quad t=1,2,3, \ldots,
$$

for some constant $C_{4}$, and therefore converges Q-quadratically to $x(\mu)$.

Proof. By (39b), we have that the first Newton step $s_{1}$ satisfies

$$
\left\|s_{1}\right\| \leq C_{1}\left\|w_{1}-x(\mu)\right\|,
$$

and so, because of the definition of $C_{0}$ in (47), the next iterate $w_{2}=w_{1}+s_{1}$ satisfies

$\left\|w_{2}-x(\mu)\right\| \leq\left\|w_{1}-x(\mu)\right\|+\left\|s_{1}\right\| \leq\left(1+C_{1}\right)\left\|w_{1}-x(\mu)\right\| \leq C_{0}\left(1+C_{1}\right) \mu^{\sigma}<C \mu^{\sigma}$.

Hence, $w_{2}$ also lies in the neighborhood (19), so we can apply (46) to obtain the following estimate for the next Newton step $s_{2}$ :

$$
\left\|s_{2}\right\| \leq 2 C_{2} C_{3} \mu^{-1}\left\|s_{1}\right\|^{2} \text {. }
$$

From (48) and (50), we have that

$$
\mu^{-1}|| s_{1} \| \leq C_{0} C_{1} \mu^{\sigma-1},
$$

so by substituting (51) and using the definition (47), we obtain

$$
\left\|s_{2}\right\| \leq 2 C_{0} C_{1} C_{2} C_{3} \mu^{\sigma-1}\left\|s_{1}\right\| \leq(1 / 4)\left\|s_{1}\right\| .
$$

Hence, for the next Newton iterate $w_{3}=w_{2}+s_{2}$, we have

$$
\begin{aligned}
& \left\|w_{3}-x(\mu)\right\| \leq\left\|w_{1}-x(\mu)\right\|+\left\|s_{1}\right\|+\left\|s_{2}\right\| \\
& \quad \leq\left\|w_{1}-x(\mu)\right\|+(5 / 4)\left\|s_{1}\right\| \leq C_{0}\left(1+(5 / 4) C_{1}\right) \mu^{\sigma}<C \mu^{\sigma},
\end{aligned}
$$

so that $w_{3}$ also lies in the neighborhood defined by (19).

The argument continues inductively. We find in general that for all $t=$ $1,2,3 \ldots$, we have that

$$
\left\|s_{t+1}\right\| \leq 2 C_{2} C_{3} \mu^{-1}|| s_{t}\left\|^{2} \leq\left(2 C_{2} C_{3} \mu^{-1} \| s_{1}||\right)\right\| s_{t}\|\leq(1 / 4)\| s_{t} \|
$$

and that

$$
\begin{aligned}
\left\|w_{t+1}-x(\mu)\right\| & \leq\left\|w_{1}-x(\mu)\right\|+\sum_{j=1}^{t}\left\|s_{j}\right\| \\
& \leq\left\|w_{1}-x(\mu)\right\|+\sum_{j=1}^{t} 4^{-(j-1)}\left\|s_{1}\right\| \\
& \leq C_{0}\left(1+(4 / 3) C_{1}\right) \mu^{\sigma}<C \mu^{\sigma},
\end{aligned}
$$

so that all Newton iterates $w_{1}, w_{2}, w_{3}, \ldots$ belong to the neighborhood (19). 
From (52), we have that $\left\|s_{t}\right\|, t=1,2, \ldots$ decreases geometrically (in fact, quadratically) to zero. Therefore, $\left\{w_{t}\right\}$ is a Cauchy sequence, so it converges, say to a point $w_{*}(\mu)$. It follows from (45) that this limit point must satisfy

$$
P_{x}\left(w_{*}(\mu) ; \mu\right)=0 .
$$

Moreover, by the second-order condition (8), we have by the choice of $\bar{\mu}$ and the discussion about the Hessian $P_{x x}(\cdot ; \mu)$ and its inverse that $P_{x x}(w ; \mu)$ is positive definite for all $w$ satisfying (19). Hence, $w_{*}(\mu)$ is a local minimizer of $P(\cdot ; \mu)$. Since $x(\mu)$ is the only local minimizer of this function in the neighborhood (19) by assumption, we must have $w_{*}(\mu)=x(\mu)$.

To prove that the convergence of $\left\{w_{t}\right\}$ to $x(\mu)$ is quadratic, we estimate the error $\left\|w_{t}-x(\mu)\right\|$ in terms of $\left\|s_{t}\right\|$. By using (52), we have for all $t=1,2,3, \ldots$ that

$$
\left\|w_{t}-x(\mu)\right\|=\left\|\sum_{j=t}^{\infty} s_{j}\right\| \leq \sum_{j=t}^{\infty}\left\|s_{j}\right\| \leq \sum_{j=t}^{\infty} 4^{-(j-1)}\left\|s_{t}\right\| \leq(4 / 3)\left\|s_{t}\right\| .
$$

Similarly, we have that

$$
\left\|w_{t}-x(\mu)\right\| \geq\left\|s_{t}\right\|-\sum_{j=t+1}^{\infty}\left\|s_{j}\right\| \geq(2 / 3)\left\|s_{t}\right\| .
$$

Hence, from (46), we have

$$
\begin{aligned}
& \left\|w_{t+1}-x(\mu)\right\| \\
& \leq(4 / 3)\left\|s_{t+1}\right\| \leq(8 / 3) C_{2} C_{3} \mu^{-1}\left\|s_{t}\right\|^{2} \leq(128 / 27) C_{2} C_{3} \mu^{-1}\left\|w_{t}-x(\mu)\right\|^{2},
\end{aligned}
$$

indicating that the convergence is Q-quadratic, as claimed.

For later reference, note from (48), (50), and (52) that we have

$$
\left\|s_{t+1}\right\| \leq\left[2 C_{0} C_{1} C_{2} C_{3}\right]^{2^{t}}\left(2 C_{2} C_{3}\right)^{-1} \mu^{2^{t}(\sigma-1)+1}, \quad t=0,1,2, \ldots,
$$

and therefore from (45) we have

$$
\begin{aligned}
& \left\|P_{x}\left(w_{t+1} ; \mu\right)\right\| \\
& \leq 2 C_{2} \mu^{-2}\left\|s_{t}\right\|^{2} \leq\left[2 C_{0} C_{1} C_{2} C_{3}\right]^{2^{t}}\left(2 C_{2} C_{3}^{2}\right)^{-1} \mu^{2^{t}(\sigma-1)}, \quad t=1,2,3, \ldots .
\end{aligned}
$$

\section{Consistency with a Newton Line-Search Strategy}

Theorem 1 describes the classic locally convergent version of Newton's method, in which the unit step is taken at each iteration. Practical versions of Newton's method use a line search or trust region to ensure convergence to a stationary point or a local minimizer. In this section, we consider a line search strategy. Under standard conditions for acceptance of the step length $\alpha$, and assuming that the current point $w$ lies in the neighborhood (19) and that the assumptions 
of Theorem 1 are satisfied, we show that $\alpha=1$ is an acceptable step length, for all $\mu$ sufficiently small. In fact, the Newton/log-barrier algorithm to be described in the next section will use a modification of the line search conditions below, but they reduce to these conditions within the neighborhood (19).

The line search conditions on $\alpha$ are as follows:

$$
\begin{aligned}
P(w+\alpha s ; \mu) & \leq P(w ; \mu)+\underline{\gamma} \alpha s^{T} P_{x}(w ; \mu) \\
\left|s^{T} P_{x}(w+\alpha s ; \mu)\right| & \leq-\bar{\gamma} s^{T} P_{x}(w ; \mu),
\end{aligned}
$$

where $\underline{\gamma}$ and $\bar{\gamma}$ are parameters that satisfy

$$
0<\underline{\gamma}<1 / 2, \quad \underline{\gamma}<\bar{\gamma}<1
$$

These conditions, which ensure that $\alpha$ is an approximate local minimizer of the function $P(\cdot ; \mu)$ along the direction $s$, can be found in Fletcher [11, (2.5.1), (2.5.6)], who shows that there exists a range of acceptable points for which they are satisfied. Naturally, we also require $w+\alpha s$ to be strictly feasible for the problem (1), since the function $P(\cdot ; \mu)$ is not defined otherwise.

A number of general techniques are available for choosing $\alpha$ to satisfy conditions (54); see, for example, Fletcher [11, Section 2.6] and the references therein. A technique that is specialized to the characteristics of the log-barrier function is described by Murray and Wright [20].

In accordance with standard practice, we accept the step $\alpha=1$ whenever this choice satisfies the conditions (54). We now verify that such is indeed the case for all $\mu$ sufficiently small, whenever $w$ satisfies (19).

We verify first condition (54b). From (44a), (39b), and using $\nabla c_{i}(w)^{T} s=$ $O\left(\left\|\hat{U}(w)^{T} s\right\|\right)$ for $i=1,2, \ldots, q$, we have that

$$
\begin{aligned}
s^{T} P_{x}(w+s ; \mu) & =\sum_{i=1}^{q} O\left(\mu^{-2}\|s\|\left\|\hat{U}(w)^{T} s\right\|\right) \nabla c_{i}(w)^{T} s+O\left(\mu^{-1}\|s\|^{3}\right) \\
& =O\left(\mu^{-2}\|s\|\left\|\hat{U}(w)^{T} s\right\|^{2}\right)+O\left(\mu^{-1}\|s\|^{3}\right) \\
& =O\left(\mu^{-1}\|s\|\right)\left[\mu^{-1}|| \hat{U}(w)^{T} s\left\|^{2}+\right\| \tilde{U}(w)^{T} s \|^{2}\right] \\
& =O\left(\mu^{\sigma-1}\right)\left[\mu^{-1}\left\|\hat{U}(w)^{T} s\right\|^{2}+\left\|\tilde{U}(w)^{T} s\right\|^{2}\right]
\end{aligned}
$$

For the directional derivative at $\alpha=0$, we use (14), (28), and the properties of $\hat{D}(w)$ to deduce that there exist constants $C_{5}>0$ and $C_{6} \geq 0$ such that the following chain of inequalities holds, for all $\mu$ sufficiently small:

$$
\begin{aligned}
- & s^{T} P_{x}(w ; s) \\
= & s^{T} P_{x x}(w ; \mu) s \\
= & {\left[\hat{U}(w)^{T} s\right]^{T} G_{11}(w)\left[\hat{U}(w)^{T} s\right]+2\left[\hat{U}(w)^{T} s\right]^{T} G_{12}(w)\left[\tilde{U}(w)^{T} s\right] } \\
& +\left[\tilde{U}(w)^{T} s\right]^{T} G_{22}(w)\left[\tilde{U}(w)^{T} s\right] \\
\geq & C_{5} \mu^{-1}\left\|\hat{U}(w)^{T} s\right\|^{2}-2 C_{6}\left\|\hat{U}(w)^{T} s\right\|\left\|\tilde{U}(w)^{T} s\right\|+C_{5}\left\|\tilde{U}(w)^{T} s\right\|^{2} \\
= & \left(C_{5} / 2\right) \mu^{-1}\left\|\hat{U}(w)^{T} s\right\|^{2}+\left(C_{5}-2 \mu C_{6}^{2} / C_{5}\right)\left\|\tilde{U}(w)^{T} s\right\|^{2}
\end{aligned}
$$




$$
\begin{aligned}
& +\left[\left(C_{5} /(2 \mu)\right)^{1 / 2}\left\|\hat{U}(w)^{T} s\right\|-\left(2 \mu / C_{5}\right)^{1 / 2} C_{6}\left\|\tilde{U}(w)^{T} s\right\|\right]^{2} \\
\geq & \left(C_{5} / 2\right)\left[\mu^{-1}\left\|\hat{U}(w)^{T} s\right\|^{2}+\left\|\tilde{U}(w)^{T} s\right\|^{2}\right] .
\end{aligned}
$$

By comparing this final expression with (56), we see that

$$
\left|s^{T} P_{x}(w+s ; \mu)\right|=O\left(\mu^{\sigma-1}\right)\left[-s^{T} P_{x}(w ; \mu)\right],
$$

so that for any fixed $\bar{\gamma} \in(0,1)$, or indeed any varying $\gamma$ such that

$$
\bar{\gamma}=\Omega\left(\mu^{\bar{\sigma}-1}\right),
$$

for some $\bar{\sigma} \in(1, \sigma)$, the condition (54b) will be satisfied whenever $\mu$ is sufficiently small.

We now verify that (54a) holds for $\alpha=1$. Since by Taylor's theorem we have

$$
P(w+s ; \mu)=P(w ; \mu)+s^{T} P_{x}(w ; \mu)+\frac{1}{2} s^{T} P_{x x}(w+\tau s ; \mu) s,
$$

for some $\tau \in(0,1)$, a sufficient condition for (54a) is that

$$
\frac{1}{2} s^{T} P_{x x}(w+\tau s ; \mu) s \leq-(1-\underline{\gamma}) s^{T} P_{x}(w ; \mu),
$$

for each $\tau \in(0,1)$. Because of (14), the expression is equivalent to

$$
\frac{1}{2} s^{T}\left[P_{x x}(w+\tau s ; \mu)-P_{x}(w ; \mu)\right] s \leq(1 / 2-\underline{\gamma}) s^{T} P_{x x}(w ; \mu) s,
$$

and by (57), a sufficient condition for this inequality to be satisfied is that

$$
\begin{aligned}
& \left|s^{T}\left[P_{x x}(w+\tau s ; \mu)-P_{x}(w ; \mu)\right] s\right| \\
& \leq C_{5}(1 / 2-\underline{\gamma})\left[\left.\mu^{-1}|| \hat{U}(w)^{T} s\right|^{2}+\left\|\tilde{U}(w)^{T} s\right\|^{2}\right] .
\end{aligned}
$$

We bound the left-hand side of (61) by taking the inner product of (40) with the vector $s$ and estimating the four terms $s^{T} r_{1}, s^{T} r_{2}, s^{T} r_{3}$, and $s^{T} r_{4}$ individually. By (42a), and using again the fact that $\nabla c_{i}(w)^{T} s=O\left(\left\|\hat{U}(w)^{T} s\right\|\right)$, we obtain

$$
\begin{aligned}
s^{T} r_{1} & =O\left(\mu^{-2}\|s\|\right)\left\|\hat{U}(w)^{T} s\right\|^{2}+O\left(\mu^{-1}\|s\|^{3}\right) \\
& =O\left(\mu^{-1}\|s\|\right)\left[\mu^{-1}\left\|\hat{U}(w)^{T} s\right\|^{2}+\left\|\tilde{U}(w)^{T} s\right\|^{2}\right] .
\end{aligned}
$$

From the definition of $r_{2}$ and (43), we have that

$$
s^{T} r_{2}=O\left(\mu^{-1}|| s \|^{3}\right)=O\left(\mu^{-1}\|s\|\right)\|s\|^{2} .
$$

For the other two terms, we have from $r_{3}=O\left(\|s\|^{2}\right)$ and $r_{4}=O\left(\mu\|s\|^{3}\right)$ that

$$
s^{T} r_{3}=O\left(\|s\|^{3}\right), \quad s^{T} r_{4}=O\left(\mu\|s\|^{4}\right) .
$$


Using (39), we have by combining (62), (63), and (64) with (40) that

$$
\left|s^{T}\left[P_{x x}(w+\tau s ; \mu)-P_{x}(w ; \mu)\right] s\right|=O\left(\mu^{\sigma-1}\right)\left[\mu^{-1}\left\|\hat{U}(w)^{T} s\right\|^{2}+\left\|\tilde{U}(w)^{T} s\right\|^{2}\right] \text {. }
$$

Because $\sigma-1>0$ and $(1 / 2-\gamma)>0$, we see that (61) and therefore (54a) is satisfied for all sufficiently small $\mu$.

We now summarize the results of this section.

Theorem 2. Suppose that the assumptions of Theorem 1 are satisfied. Then given any constants $\gamma$ and $\bar{\gamma}$ satisfying (55), there exists $\bar{\mu}_{1} \in\left(0, \bar{\mu}_{0}\right]$ such that when $w_{1}$ is any point satisfying (48) with $\mu \in\left(0, \bar{\mu}_{1}\right]$, the full step $\alpha=1$ along the Newton direction $s_{1}$ will satisfy the tests (54).

\section{Rapid Convergence When the Objective Function Is Linear}

We now discuss the case in which the objective function $f(\cdot)$ in (1) is linear (having noted in Section 2 that any nonlinear program can be reformulated in this fashion). We outline a version of the Newton/log-barrier method and show that it converges rapidly for certain choices of the parameters and certain line search strategies. We assume throughout that there is at least one active constraint (that is, $q \geq 1$ ), since the other case is trivial in the current context.

We start with a result that describes the first Newton step taken immediately after a reduction in the barrier parameter. In particular, we examine the point obtained by applying a line search along the Newton direction together with a stopping criterion based on the directional derivative, similar to (54b). For convenience, we use a scaled version of the line search parameter, replacing $\alpha$ in (54) by $\bar{\alpha}\left(\mu / \mu_{-}\right)$.

Theorem 3. Suppose that $f$ is linear (that is, $\nabla f(x) \equiv g$ ) and that the barrier parameter values $\mu_{-}$and $\mu$ satisfy the condition

$$
\mu \in\left[\rho_{0} \mu_{-}^{\tilde{\sigma}}, \rho_{1} \mu_{-}\right],
$$

where $\rho_{0}>0, \rho_{1} \in(0,1)$, and $\tilde{\sigma} \in(1,2]$ are constants. Suppose too that the bound

$$
\left\|P_{x}\left(x ; \mu_{-}\right)\right\| \leq \mu_{-}
$$

is satisfied at the current value of $x$. Then if $s$ is the Newton direction for $P(\cdot ; \mu)$ from $x$, we have that

$$
\begin{aligned}
& -s^{T} P_{x}\left(x+\bar{\alpha}\left(\mu / \mu_{-}\right) s ; \mu\right) \\
& =\frac{\mu_{-}^{2}}{\mu}\left[1-\frac{\mu}{\mu_{-}}+O\left(\mu_{-}\right)\right] \sum_{i=1}^{q} \frac{c_{i}(x)}{c_{i}\left(x+\bar{\alpha}\left(\mu / \mu_{-}\right) s\right)}\left[(1-\bar{\alpha})\left(1-\frac{\mu}{\mu_{-}}\right)+O\left(\mu_{-}\right)\right] \\
& \quad+O\left(\mu_{-}^{2}\right) \\
& =\frac{\mu_{-}^{2}}{\mu}\left[1-\frac{\mu}{\mu_{-}}+O\left(\mu_{-}\right)\right] \sum_{i=1}^{q}\left[\frac{(1-\bar{\alpha})\left(1-\mu / \mu_{-}\right)+O\left(\mu_{-}\right)}{(1-\bar{\alpha})\left(1-\mu / \mu_{-}\right)+\mu / \mu_{-}+O\left(\mu_{-}\right)}\right] \\
& \quad+O\left(\mu_{-}^{2}\right) .
\end{aligned}
$$


Proof. The proof from the analysis in the proof of Theorem 2 in S. Wright and Jarre [29], allowing for the changes of notation ( $s$ in our analysis replaces $p$ in [29], $\mu_{-}$replaces $\mu, \mu$ replaces $\mu_{+}$, and $\bar{\alpha}$ replaces $\tau$ ). Note that our condition (66) is obtained by setting $\sigma=1$ in [29, eq. (30)].

From (13a), we have that

$$
-s^{T} P_{x}\left(x+\bar{\alpha}\left(\mu / \mu_{-}\right) s ; \mu\right)=-s^{T} g+\mu \sum_{i=1}^{m} \frac{s^{T} \nabla c_{i}\left(x+\bar{\alpha}\left(\mu / \mu_{-}\right) s\right)}{c_{i}\left(x+\bar{\alpha}\left(\mu / \mu_{-}\right) s\right)} .
$$

From [29, eq. (47)], we have

$$
s^{T} g=-q\left(\mu_{-}^{2} / \mu\right)\left[1-\mu / \mu_{-}+O\left(\mu_{-}\right)\right] .
$$

We have from the display equation following [29, eq. (47)] that

$$
\begin{aligned}
& s^{T} \nabla c_{i}\left(x+\bar{\alpha}\left(\mu / \mu_{-}\right) s\right) \\
& =-\left(\mu_{-}^{2} / \mu\right) \lambda_{i}\left(x, \mu_{-}\right)^{-1}\left[1-\mu / \mu_{-}+O\left(\mu_{-}\right)\right], \quad i=1,2, \ldots, q,
\end{aligned}
$$

where $\lambda_{i}\left(x, \mu_{-}\right)=\mu_{-} / c_{i}(x)$ as defined in (11). Similarly, we have from [29, eq. (41)] and (11) that

$$
\begin{aligned}
c_{i}\left(x+\bar{\alpha}\left(\mu / \mu_{-}\right) s\right) & =-\mu_{-} \lambda_{i}\left(x, \mu_{-}\right)^{-1}\left[1-\bar{\alpha}\left(1-\mu / \mu_{-}\right)+O\left(\mu_{-}\right)\right] \\
& =c_{i}(x)\left[1-\bar{\alpha}\left(1-\mu / \mu_{-}\right)+O\left(\mu_{-}\right)\right], \quad i=1,2, \ldots, q .
\end{aligned}
$$

For an estimate of $\|s\|$, we have from [29, eq. (33)] that

$$
\|s\|=O\left(\mu_{-}^{2} / \mu\right)
$$

For the inactive indices $i=q+1, \ldots, m$, we note that $c_{i}\left(x+\bar{\alpha}\left(\mu / \mu_{-}\right) s\right)$ is uniformly bounded away from zero, so from (73) we have

$$
\frac{s^{T} \nabla c_{i}\left(x+\bar{\alpha}\left(\mu / \mu_{-}\right) s\right)}{c_{i}\left(x+\bar{\alpha}\left(\mu / \mu_{-}\right) s\right)}=O(\|s\|)=O\left(\mu_{-}^{2} / \mu\right), \quad i=q+1, \ldots, m .
$$

We now substitute (11), (70), (71), (72), (73), and (74) into (69) to obtain

$$
\begin{aligned}
& -s^{T} P_{x}\left(x+\bar{\alpha}\left(\mu / \mu_{-}\right) s ; \mu\right) \\
& =\frac{\mu_{-}^{2}}{\mu}\left[1-\mu / \mu_{-}+O\left(\mu_{-}\right)\right] \sum_{i=1}^{q}\left[1-\frac{\lambda_{i}\left(x, \mu_{-}\right)^{-1} \mu}{c_{i}\left(x+\bar{\alpha}\left(\mu / \mu_{-}\right) s\right)}\right]+O\left(\mu_{-}^{2}\right) \\
& =\frac{\mu_{-}^{2}}{\mu}\left[1-\mu / \mu_{-}+O\left(\mu_{-}\right)\right] \sum_{i=1}^{q} \frac{c_{i}\left(x+\bar{\alpha}\left(\mu / \mu_{-}\right) s\right)-\lambda_{i}\left(x, \mu_{-}\right)^{-1} \mu}{c_{i}\left(x+\bar{\alpha}\left(\mu / \mu_{-}\right) s\right)}+O\left(\mu_{-}^{2}\right) \\
& =\frac{\mu_{-}^{2}}{\mu}\left[1-\mu / \mu_{-}+O\left(\mu_{-}\right)\right] \sum_{i=1}^{q} c_{i}(x) \frac{1-\bar{\alpha}\left(1-\mu / \mu_{-}\right)+O\left(\mu_{-}\right)-\mu / \mu_{-}}{c_{i}\left(x+\bar{\alpha}\left(\mu / \mu_{-}\right) s\right)}+O\left(\mu_{-}^{2}\right),
\end{aligned}
$$

giving (67). 
To prove the other estimate (68), we use (72) and (11) to obtain

$$
\begin{aligned}
\frac{c_{i}(x)}{c_{i}\left(x+\bar{\alpha}\left(\mu / \mu_{-}\right) s\right)} & =\frac{c_{i}(x)}{-\mu_{-} \lambda_{i}\left(x, \mu_{-}\right)^{-1}\left[1-\bar{\alpha}\left(1-\mu / \mu_{-}\right)+O\left(\mu_{-}\right)\right]} \\
& =\frac{1}{(1-\bar{\alpha})\left(1-\mu / \mu_{-}\right)+\mu / \mu_{-}+O\left(\mu_{-}\right)} .
\end{aligned}
$$

The result follows by substitution into (67). (Note that (68) is also an immediate consequence of [29, eq. (48)].)

We next show that when we apply a condition similar to (54b) to the first Newton step immediately after reduction from $\mu_{-}$to $\mu$, the step lengths satisfying this condition form a small interval around $\bar{\alpha}=1$.

Theorem 4. Suppose that the conditions of Theorem 3 are satisfied, in particular that $x, s, \mu$, and $\mu_{-}$have the properties specified therein. Let $\breve{\sigma}$ be a constant satisfying $\tilde{\sigma}<\breve{\sigma}<2$. We then have

$$
-s^{T} P_{x}(x ; \mu)=\Theta\left(\mu_{-}^{2} / \mu\right) .
$$

Moreover, a directional derivative condition of the form

$$
\left|s^{T} P_{x}\left(x+\bar{\alpha}\left(\mu / \mu_{-}\right) s ; \mu\right)\right| \leq-C_{10}\left(\mu_{-}^{\breve{\sigma}} / \mu\right) s^{T} P_{x}(x ; \mu),
$$

or alternatively

$$
\left|s^{T} P_{x}\left(x+\bar{\alpha}\left(\mu / \mu_{-}\right) s ; \mu\right)\right| \leq C_{11} \mu_{-}^{\breve{\sigma}+2} / \mu^{2},
$$

where $C_{10}$ and $C_{11}$ are given positive constants, is satisfied if and only if

$$
|1-\bar{\alpha}|=O\left(\mu_{-}^{\breve{\sigma}-1}\right) .
$$

Proof. We prove (75) by substituting $\bar{\alpha}=0$ into (68). We obtain

$$
\begin{aligned}
-s^{T} P_{x}(x ; \mu) & =\frac{\mu_{-}^{2}}{\mu}\left(1-\mu / \mu_{-}\right) \sum_{i=1}^{q}\left[\frac{1-\mu / \mu_{-}+O\left(\mu_{-}\right)}{1+O\left(\mu_{-}\right)}\right]+O\left(\mu_{-}^{2}\right) \\
& =q \frac{\mu_{-}^{2}}{\mu}\left(1-\mu / \mu_{-}\right)\left(1-\mu / \mu_{-}+O\left(\mu_{-}\right)\right)+O\left(\mu_{-}^{2}\right) \\
& =q \frac{\mu_{-}^{2}}{\mu}\left(1-\mu / \mu_{-}\right)^{2}+O\left(\mu_{-}^{3} / \mu\right),
\end{aligned}
$$

where in the third equality we used the relations $\mu<\mu_{-} \ll 1$. It follows directly from $0<\mu<\mu_{-}$that

$$
-s^{T} P_{x}(x ; \mu) \leq q \frac{\mu_{-}^{2}}{\mu}+O\left(\mu_{-}^{3} / \mu\right)
$$

Since $\mu \leq \rho_{1} \mu_{-}$, we have $1-\mu / \mu_{-} \geq 1-\rho_{1}$, so it follows from (79) that

$$
-s^{T} P_{x}(x ; \mu) \geq q \frac{\mu_{-}^{2}}{\mu}\left(1-\rho_{1}\right)^{2}+O\left(\mu_{-}^{3} / \mu\right) .
$$


By taking $\mu_{-}$sufficiently small, the claim (75) follows immediately from (80) and (81).

We now prove the second statement for (77); the other case involving (76) follows immediately from (75).

We first prove the reverse implication; that is, we assume that (78) is satisfied and prove that (77) holds. We start by estimating the various terms that appear in $(68)$. Because of $(65)$, we have

$$
\mu_{-} \leq\left(\mu / \rho_{0}\right)^{1 / \tilde{\sigma}}
$$

Using this condition, together with $\breve{\sigma}>\tilde{\sigma}$, we have

$|1-\bar{\alpha}|\left(1-\mu / \mu_{-}\right)=O\left(\mu_{-}^{\breve{\sigma}-1}\right)=O\left(\mu_{-}^{\breve{\sigma}} / \mu\right)\left(\mu / \mu_{-}\right)=O\left(\mu^{\breve{\sigma} / \tilde{\sigma}-1}\right)\left(\mu / \mu_{-}\right)=o(1)\left(\mu / \mu_{-}\right)$.

Using (82) again, we also have

$$
\mu_{-}=\left(\mu_{-}^{2} / \mu\right)\left(\mu / \mu_{-}\right)=O\left(\mu^{2 / \tilde{\sigma}-1}\right)\left(\mu / \mu_{-}\right)=o(1)\left(\mu / \mu_{-}\right) .
$$

It follows from these estimates that each term in the summation in (68) satisfies

$$
\frac{(1-\bar{\alpha})\left(1-\mu / \mu_{-}\right)+O\left(\mu_{-}\right)}{(1-\bar{\alpha})\left(1-\mu / \mu_{-}\right)+\mu / \mu_{-}+O\left(\mu_{-}\right)}=\frac{O\left(\mu_{-}^{\breve{\sigma}-1}\right)}{(1+o(1))\left(\mu / \mu_{-}\right)}=O\left(\mu_{-}^{\breve{\sigma}} / \mu\right) .
$$

Therefore we have from (68) that

$$
\begin{aligned}
-s^{T} P_{x}\left(x+\bar{\alpha}\left(\mu / \mu_{-}\right) s ; \mu\right) & =\left(\mu_{-}^{2} / \mu\right)\left[1-\mu / \mu_{-}+O\left(\mu_{-}\right)\right] O\left(\mu_{-}^{\breve{\sigma}} / \mu\right)+O\left(\mu_{-}^{2}\right) \\
& =O\left(\mu_{-}^{\breve{\sigma}+2} / \mu^{2}\right)+O\left(\mu_{-}^{2}\right) .
\end{aligned}
$$

The second term in dominated by the first term in this expression because from $\mu<\mu_{-}$and $\breve{\sigma}<2$, we have

$$
\mu_{-}^{2}<\frac{\mu_{-}^{4}}{\mu^{2}}=\frac{\mu_{-}^{2+\breve{\sigma}}}{\mu^{2}} \mu_{-}^{2-\breve{\sigma}} \leq \frac{\mu_{-}^{2+\breve{\sigma}}}{\mu^{2}}
$$

for all $\mu_{-}$(hence, $\mu$ ) sufficiently small. We conclude that (78) implies (77).

Now assume that (77) holds. We prove the required implication (78) by using the estimates (67) and (68). When $|1-\bar{\alpha}|=O\left(\mu_{-}\right)$, then (78) holds trivially, since $\breve{\sigma} \in(1,2)$, so we assume from here onward that this relation does not hold. We then have that

$$
|1-\bar{\alpha}|\left(1-\mu / \mu_{-}\right) \gg \mu_{-},
$$

for all $\mu_{-}$sufficiently small. Using (65), we obtain

$$
\left|(1-\bar{\alpha})\left(1-\mu / \mu_{-}\right)+O\left(\mu_{-}\right)\right| \geq \frac{1}{2}|1-\bar{\alpha}|\left(1-\mu / \mu_{-}\right) \geq \frac{1}{2}\left(1-\rho_{1}\right)|1-\bar{\alpha}| .
$$

Likewise, we have for all $\mu_{-}$sufficiently small that

$$
1-\mu / \mu_{-}+O\left(\mu_{-}\right) \geq \frac{1}{2}\left(1-\rho_{1}\right) .
$$


In addition, we have $c_{i}(x)>0$, and for all $\alpha$ of interest we also have that $c_{i}\left(x+\bar{\alpha}\left(\mu / \mu_{-}\right) s\right)>0$. Hence, by appealing to (67), we have from (77) that

$$
\frac{\mu_{-}^{2}}{\mu} \frac{1}{4}\left(1-\rho_{1}\right)^{2}|1-\bar{\alpha}| \sum_{i=1}^{q} \frac{c_{i}(x)}{c_{i}\left(x+\bar{\alpha}\left(\mu / \mu_{-}\right) s\right)} \leq C_{11} \frac{\mu_{-}^{\breve{\sigma}+2}}{\mu^{2}} .
$$

Since all terms in the summation are positive, we have for each $i=1,2, \ldots, q$ that

$$
\frac{\mu_{-}^{2}}{\mu} \frac{1}{4}\left(1-\rho_{1}\right)^{2}|1-\bar{\alpha}| \frac{c_{i}(x)}{c_{i}\left(x+\bar{\alpha}\left(\mu / \mu_{-}\right) s\right)} \leq C_{11} \frac{\mu_{-}^{\breve{\sigma}+2}}{\mu^{2}} .
$$

By defining $\bar{C}_{11}=4\left(1-\rho_{1}\right)^{-2} C_{11}$, we can rearrange this equation and use (72) to obtain

$$
\begin{aligned}
|1-\bar{\alpha}| & \leq \bar{C}_{11} \frac{c_{i}\left(x+\bar{\alpha}\left(\mu / \mu_{-}\right) s\right)}{c_{i}(x)} \frac{\mu_{-}^{\breve{\sigma}}}{\mu} \\
& =\bar{C}_{11}\left[1-\bar{\alpha}\left(1-\mu / \mu_{-}\right)+O\left(\mu_{-}\right)\right] \frac{\mu_{-}^{\breve{\sigma}}}{\mu} .
\end{aligned}
$$

By further rearrangement, we obtain

$$
|1-\bar{\alpha}|\left[1-\bar{C}_{11} \frac{\mu_{-}^{\breve{\sigma}}}{\mu}\right] \leq \bar{C}_{11} \bar{\alpha} \mu_{-}^{\breve{\sigma}-1}+O\left(\mu_{-}^{\breve{\sigma}+1} / \mu\right) \leq 3 \bar{C}_{11} \mu_{-}^{\breve{\sigma}-1},
$$

since it follows readily from (72) and the requirement that $c_{i}>0$ that $\bar{\alpha} \leq 2$, while the remainder term $O\left(\mu_{-}^{\breve{\sigma}+1} / \mu\right)$ is dominated by $\bar{C}_{11} \mu_{-}^{\breve{\sigma}-1}$ for all $\mu_{-}$(hence, $\mu$ ) sufficiently small. From (82), and the assumption of $1<\tilde{\sigma}<\breve{\sigma}$, we have that

$$
\bar{C}_{11} \frac{\mu_{-}^{\breve{\sigma}}}{\mu} \leq \bar{C}_{11} / \rho_{0}^{1 / \tilde{\sigma}} \mu^{\breve{\sigma} / \tilde{\sigma}-1} \leq \frac{1}{2},
$$

for all $\mu_{-}$sufficiently small. By substituting this bound into (83) we obtain the required estimate (78).

It follows from Theorem 4 that the step $\bar{\alpha}=1$, or equivalently $\alpha=\mu / \mu_{-}$, is nearly optimal at this first step after reduction of $\mu$. We show now that all steplengths satisfying (78) yield iterates that lie within the neighborhod (19), for a certain choice of $\sigma$.

Theorem 5. Suppose that the conditions of Theorem \& are satisfied and that the step $\alpha=\bar{\alpha}\left(\mu / \mu_{-}\right)$taken along the direction s satisfies (78). We then have that

$$
\left\|x+\bar{\alpha}\left(\mu / \mu_{-}\right) s-x(\mu)\right\|=O\left(\mu_{-}^{\breve{\sigma}}\right)=O\left(\mu^{\breve{\sigma} / \tilde{\sigma}}\right) .
$$

Proof. Note first that

$$
\left\|x+\bar{\alpha}\left(\mu / \mu_{-}\right) s-x(\mu)\right\| \leq\left\|x+\left(\mu / \mu_{-}\right) s-x(\mu)\right\|+|\bar{\alpha}-1|\left\|\left(\mu / \mu_{-}\right) s\right\| .
$$

From Wright and Jarre [29, Theorem 1], and using (66), we have that

$$
\left\|x+\left(\mu / \mu_{-}\right) s-x(\mu)\right\|=O\left(\mu_{-}^{2}\right) .
$$


From [29, eqs. (32), (33)], and noting that our current results use $\sigma=2$ in [29, eq. (33)], we have that

$$
\left\|\left(\mu / \mu_{-}\right) s\right\|=\Theta\left(\mu_{-}\right) .
$$

The first equality in (84) is obtained by substituting (86), (87), and (78) into (85). The second equality follows from (82).

We now specify Algorithm NLB, our variant of the Newton/log-barrier method. Since we are interested only in local behavior of the method, we assume that the initial value $\mu_{0}$ is already small enough that the range of values in (65) is nonempty for all $\mu_{-} \leq \mu_{0}$. Simple modifications can be applied to make this method valid globally.

\section{Algorithm NLB}

Given $\mu_{0}>0, \rho_{0} \in(0,1), \rho_{1} \in(0,1)$, and $\tilde{\sigma}, \breve{\sigma}$ with $1<\tilde{\sigma}<\breve{\sigma}<2$;

Set $k \leftarrow 0, x_{-1} \leftarrow$ strictly feasible initial value;

Set tol1 $\leftarrow \infty$;

repeat

$x_{k} \leftarrow \operatorname{Newton}\left(x_{k-1}, \mu_{k}\right.$, tol1);

if $\mu_{k}$ sufficiently small

terminate;

Set $k \leftarrow k+1$;

Choose $\mu_{k} \in\left[\rho_{0} \mu_{k-1}^{\tilde{\sigma}}, \rho_{1} \mu_{k-1}\right]$;

Set tol $\leftarrow \leftarrow \mu_{k-1}^{\breve{\sigma}+2} / \mu_{k}^{2}$;

end (repeat)

The Newton procedure with line search is defined as follows

Procedure Newton $(x, \mu$, tol1);

Given $\underline{\gamma}$ and $\bar{\gamma}$ satisfying (55);

Set $t \leftarrow 0, w_{0} \leftarrow x$;

while $\left\|P_{x}\left(w_{t} ; \mu\right)\right\|>\mu$

Set $s_{t} \leftarrow-P_{x x}\left(w_{t} ; \mu\right)^{-1} P_{x}\left(w_{t} ; \mu\right)$;

Choose $\alpha$ such that (54a) and

$$
\left|s_{t}^{T} P_{x}\left(w_{t}+\alpha s_{t} ; \mu\right)\right| \leq \min \left(\text { tol1 },-\bar{\gamma} s_{t}^{T} P_{x}\left(w_{t} ; \mu\right)\right),
$$

are satisfied, accepting $\alpha=1$ if it satisfies these conditions;

Set $w_{t+1} \leftarrow w_{t}+\alpha s_{t}$;

Set $t \leftarrow t+1$;

end (while)

return $w_{t}$.

We now show that in the line-search criterion (88), the right-hand side is equal to tol1 at the first iteration of each call to Procedure Newton, while the alternative value $-\bar{\gamma} s_{0}^{T} P_{x}\left(w_{0} ; \mu\right)$ is operative on subsequent calls.

Theorem 6. Suppose that $f$ is linear. Then for all steps $k$ in Algorithm NLB with $k>0$ sufficiently large, the following properties are satisfied. 
(i) At the first step $(t=0)$ taken within Procedure Newton within iteration $k$ of Algorithm NLB, we have that tol $1<-\bar{\gamma} s_{0}^{T} P_{x}\left(w_{0} ; \mu\right)$, so the value $\alpha=\bar{\alpha}\left(\mu_{k} / \mu_{k-1}\right)$ selected at this step will satisfy (78), for $\mu_{-}=\mu_{k-1}$.

(ii) At the second and subsequent steps $(t=1,2,3, \ldots)$ within Procedure Newton at iteration $k$, we have that tol $1>-\bar{\gamma} s_{t}^{T} P_{x}\left(w_{t} ; \mu\right)$. Moreover, $\alpha=1$ is the chosen step length at each of these iterations.

Proof. Using the notation $\mu_{-}=\mu_{k-1}$ and $\mu=\mu_{k}$, we have from (82), (75), and $\breve{\sigma}>\tilde{\sigma}$ that

$$
\mathrm{t} 011 \triangleq \frac{\mu_{-}^{\breve{\sigma}+2}}{\mu^{2}}=\frac{\mu_{-}^{2}}{\mu} \frac{\mu_{-}^{\breve{\sigma}}}{\mu} \leq \frac{\mu_{-}^{2}}{\mu} \rho_{0}^{-\breve{\sigma} / \tilde{\sigma}} \mu^{\breve{\sigma} / \tilde{\sigma}-1}<-\bar{\gamma} s^{T} P_{x}(x ; \mu),
$$

for all $\mu$ sufficiently small. It follows from (88) that (77) is satisfied for $C_{11}=1$. Because of the convergence criterion at the previous invocation of Procedure Newton, we have that $\left\|P_{x}\left(w_{0} ; \mu_{-}\right)\right\| \leq \mu_{-}$, so that condition (66) is satisfied. The other conditions of Theorem 3 (and Theorem 4) also hold, so by the forward implication in Theorem 4, we conclude that (78) holds, proving (i).

Since (78) is satisfied by the first steplength in Procedure Newton, and the other conditions of Theorem 4 are satisfied, we have from Theorem 5 that the new iterate

$$
w_{1} \triangleq x+\bar{\alpha}\left(\mu / \mu_{-}\right) s
$$

will satisfy

$$
\left\|w_{1}-x(\mu)\right\|=O\left(\mu^{\breve{\sigma} / \tilde{\sigma}}\right)
$$

By choosing some value

$$
\sigma \in(1, \breve{\sigma} / \tilde{\sigma})
$$

and choosing $k$ large enough that $\mu_{k}$ is sufficiently small, we have from Theorem 2 that the tests (54) are satisfied by all subsequent iterates of the unit-step Newton's method. Moreover, by a simple inductive argument based on (49), we have that $\left\|w_{t+1}-x(\mu)\right\| \leq\left\|w_{t}-x(\mu)\right\|$ for $t=1,2,3, \ldots$, where $\left\{w_{t}\right\}$ is the sequence of iterates generated by the unit-step Newton's method, so that we have

$$
\|w-x(\mu)\|=O\left(\mu^{\breve{\sigma} / \tilde{\sigma}}\right),
$$

for $w=w_{t}, t=1,2, \ldots$

By showing that tol1 $>-\bar{\gamma} s^{T} P_{x}(w ; \mu)$ for all $w$ satisfying (90), we ensure that the condition (88) is identical to $(54 \mathrm{~b})$ at each iterate, so that the unit step length is in fact accepted in Procedure Newton for $t=2,3, \ldots$, and hence our claim (ii) holds. The following argument suffices: By using (14), (39b) with $\sigma=\breve{\sigma} / \tilde{\sigma}$, and the estimate (29), we have that

$$
\begin{aligned}
& -\bar{\gamma} s^{T} P_{x}(w ; \mu) \\
& =\bar{\gamma} s^{T} P_{x x}(w ; \mu) s \leq O\left(\|s\|^{2}\right) O\left(\left\|P_{x x}(w ; \mu)\right\|\right)=O\left(\mu^{2 \breve{\sigma} / \tilde{\sigma}-1}\right)=o(1) \mu^{\breve{\sigma}} .
\end{aligned}
$$


Meanwhile from (65), we have

$$
\text { tol1 }=\frac{\mu_{-}^{\breve{\sigma}+2}}{\mu^{2}} \geq \frac{\left(\mu / \rho_{1}\right)^{\breve{\sigma}+2}}{\mu^{2}}=\Theta\left(\mu^{\breve{\sigma}}\right) .
$$

The desired inequality follows by comparing these last two expressions.

Part (i) of Theorem 5 is predicated on the existence of an $\alpha$ satisfying both (54a) and (88). We now prove existence by showing that the particular choice $\bar{\alpha}=1$ (corresponding to $\alpha=\mu_{k} / \mu_{k-1}$ ) satisfies these two line-search conditions. It follows that Procedure Newton is well defined for all $\mu_{k}$ sufficiently small.

Theorem 7. Suppose that $f$ is linear. Then for all $k$ sufficiently large, at the first step taken at each invocation of Procedure Newton $(t=0)$, there exists a value of $\alpha$ that satisfies (54a) and (88).

Proof. Theorem 4 shows that there exists a range of values of $\bar{\alpha}$ satisfying (78) for which (77) is satisfied, for any given $C_{11}>0$. Noting that Theorem 6 (i) shows that the right-hand side of (88) is $\mu_{k-1}^{\breve{\sigma}+2} / \mu_{k}^{2}$ at the first step $(t=0)$ of Procedure Newton, and taking $C_{11}=1$, we have that (88) is satisfied for all $\bar{\alpha}$ sufficiently close to 1 . Therefore, to prove the result, it is sufficient to show that the other line-search condition (54a) is satisfied for $\bar{\alpha}=1$.

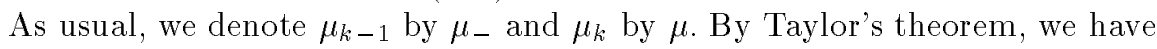

$$
P\left(x+\left(\mu / \mu_{-}\right) s ; \mu\right)-P(x ; \mu)=\frac{\mu}{\mu_{-}} \int_{0}^{1} s^{T} P_{x}\left(x+\hat{\alpha}\left(\mu / \mu_{-}\right) s ; \mu\right) d \hat{\alpha} .
$$

A cruder version of the estimate (68) suffices for the purposes of this proof. Since the summation in (68) is clearly bounded, and since $\mu_{-}^{2} / \mu=o(1)$, we can write

$$
\begin{aligned}
& -s^{T} P_{x}\left(x+\bar{\alpha}\left(\mu / \mu_{-}\right) s ; \mu\right) \\
& =\frac{\mu_{-}^{2}}{\mu}\left[1-\frac{\mu}{\mu_{-}}\right] \sum_{i=1}^{q}\left[\frac{(1-\bar{\alpha})\left(1-\mu / \mu_{-}\right)+O\left(\mu_{-}\right)}{(1-\bar{\alpha})\left(1-\mu / \mu_{-}\right)+\mu / \mu_{-}+O\left(\mu_{-}\right)}\right]+o\left(\mu_{-}\right) .
\end{aligned}
$$

By substituting $\bar{\alpha}=\hat{\alpha}$ into (92), we obtain

$$
\begin{aligned}
& \left(\mu / \mu_{-}\right) s^{T} P_{x}\left(x+\hat{\alpha}\left(\mu / \mu_{-}\right) s ; \mu\right) \\
& =-\mu_{-}\left[1-\frac{\mu}{\mu_{-}}\right] \sum_{i=1}^{q}\left[\frac{(1-\hat{\alpha})\left(1-\mu / \mu_{-}\right)+O\left(\mu_{-}\right)}{(1-\hat{\alpha})\left(1-\mu / \mu_{-}\right)+\mu / \mu_{-}+O\left(\mu_{-}\right)}\right]+o\left(\mu_{-}\right) \\
& \leq-\mu_{-}\left[1-\frac{\mu}{\mu_{-}}\right] \sum_{i=1}^{q}\left[\frac{(1-\hat{\alpha})\left(1-\mu / \mu_{-}\right)+O\left(\mu_{-}\right)}{\left(1-\mu / \mu_{-}\right)+\mu / \mu_{-}+O\left(\mu_{-}\right)}\right]+o\left(\mu_{-}\right) \\
& =-\mu_{-}\left[1-\frac{\mu}{\mu_{-}}\right]^{2} q(1-\hat{\alpha})+o\left(\mu_{-}\right) .
\end{aligned}
$$

By integrating we therefore obtain

$$
\frac{\mu}{\mu_{-}} \int_{0}^{1} s^{T} P_{x}\left(x+\hat{\alpha}\left(\mu / \mu_{-}\right) s ; \mu\right) d \hat{\alpha} \leq-\frac{1}{2} \mu_{-}\left[1-\frac{\mu}{\mu_{-}}\right]^{2} q+o\left(\mu_{-}\right) .
$$


By substituting $\bar{\alpha}=0$ into (92), we obtain

$$
\begin{aligned}
\frac{\mu}{\mu_{-}} s^{T} P_{x}(x ; \mu) & =-\mu_{-}\left[1-\frac{\mu}{\mu_{-}}\right] \sum_{i=1}^{q} \frac{1-\mu / \mu_{-}+O\left(\mu_{-}\right)}{1+O\left(\mu_{-}\right)}+o\left(\mu_{-}\right) \\
& =-\mu_{-}\left[1-\frac{\mu}{\mu_{-}}\right]^{2} q+o\left(\mu_{-}\right) .
\end{aligned}
$$

Since the first terms on the right-hand sides of both (93) and (94) have size $\Theta\left(\mu_{-}\right)$, we have

$$
\frac{\mu}{\mu_{-}} \int_{0}^{1} s^{T} P_{x}\left(x+\hat{\alpha}\left(\mu / \mu_{-}\right) s ; \mu\right) d \hat{\alpha} \leq \underline{\gamma} \frac{\mu}{\mu_{-}} s^{T} P_{x}(x ; \mu),
$$

for any $\gamma \in(0,1 / 2)$ and all $\mu_{-}$sufficiently small. By combining this bound with (91), we conclude that (54a) holds for $\bar{\alpha}=1$ (that is, $\alpha=\mu / \mu_{-}$), as required.

The proof of Theorems 6 and 7 suggest a version of Algorithm NLB that achieves superlinear convergence. Suppose we take $\mu_{k}=\rho_{0} \mu_{k-1}^{\tilde{\sigma}}$ for all $k$ sufficiently large and make the particular choice $\alpha=\mu_{k} / \mu_{k-1}$ at the first step taken at each invocation of Procedure Newton. Then the proofs of these theorems indicate that this value of $\alpha$ is accepted by the line search conditions at the first iteration of Procedure Newton, that $\alpha=1$ is accepted on subsequent steps, and that the method of Procedure Newton converges quadratically to $x\left(\mu_{k}\right)$. In fact, Procedure Newton will satisfy the condition $\left\|P_{x}\left(w_{t} ; \mu_{k}\right)\right\| \leq \mu_{k}$ and therefore will terminate after a very modest number of Newton iterations. As an example, using (49), (82) and (86), we have that

$$
\left\|w_{2}-x\left(\mu_{k}\right)\right\|=O\left(\mu_{k}^{-1}\right)\left\|w_{1}-x\left(\mu_{k}\right)\right\|^{2}=O\left(\mu_{k}^{-1} \mu_{k-1}^{2}\right)=O\left(\mu_{k}^{4 / \tilde{\sigma}-1}\right) .
$$

If $\tilde{\sigma} \in(1,4 / 3)$, we have $\left\|w_{2}-x\left(\mu_{k}\right)\right\|=o\left(\mu_{k}^{2}\right)$, and therefore from (37) it follows that $\left\|P_{x}\left(w_{2} ; \mu_{k}\right)\right\|=o\left(\mu_{k}\right)$, so that for this choice of $\tilde{\sigma}$, the convergence tolerance is satisfied after just two Newton steps. (Values $\tilde{\sigma} \geq 4 / 3$ can be accommodated by using additional Newton steps.) Overall superlinear convergence of Algorithm NLB to $x^{*}$ follows from the fact that the work per invocation of Procedure Newton is bounded, and the sequence $\left\{\mu_{k}\right\}$ converges superlinearly to zero.

As pointed out by a referee, the search direction and special steplength $\mu_{k} / \mu_{k-1}$ used by this variant of Algorithm NLB produce an identical result to the extrapolation step of Benchakroun, Dussault, and Mansouri [1]. The fact that one additional Newton step suffices for each value of $\mu$, provided that $\tilde{\sigma} \in(1,4 / 3)$ is also proved by these authors. Conn, Gould, and Toint [5] have a similar result for their acceleration technique. What is perhaps surprising about the approach described in this paper is that the same result can be recovered without the use of special search directions. When the objective is linear, the usual Newton step for $P(\cdot ; \mu)$ has the required properties.

Even if we do not make the special choice $\alpha=\mu_{k} / \mu_{k-1}$ for the first iteration of Procedure Newton, but instead rely on the line search procedure and the conditions (54a) and (88) to identify an appropriate value of $\alpha$ at this step, 
the overall rate will remain superlinear provided that the number of function and derivative evaluations required to identify the first $\alpha$ values is bounded. The latter requirement can be expected to hold for all reasonable line-search algorithms. For instance, the specialized line search procedure for log-barrier functions, such as that of Murray and Wright [20] can be expected to find an appropriate $\alpha$ with just a couple of evaluations of $P\left(\cdot ; \mu_{k}\right)$ and $P_{x}\left(\cdot ; \mu_{k}\right)$.

\section{Discussion}

We conclude by expanding the discussion of Section 2 to note a few more points about the context of this work.

A consequence of the ill conditioning of $P_{x x}(x ; \mu)$ is that standard methods of linear algebra for obtaining $s$, which solve the system

$$
P_{x x}(x ; \mu) s=-P_{x}(x ; \mu),
$$

may be inefficient or inaccurate. A number of authors have proposed alternative formulations of systems such as this (Gould [14]) or modified factorization schemes (Broyden and Attia [2], M. Wright [25]) or have shown that the inaccuracy introduced by the Cholesky factorization may not be especially detrimental (M. Wright [27], S. Wright [28]). Since these numerical considerations are outside the scope of this paper, we omit further discussion of this point.

Another line of investigation in the literature that is relevant to this paper concerns the analytic center method for certain types of convex programming problems. In this method, a logarithmic potential function different from $P(\cdot ; \mu)$ is used, typically having the form

$$
\phi(x ; \eta)=-\omega \log (\eta-f(x))-\sum_{i=1}^{m} \log c_{i}(x) .
$$

where $\omega>0$ is some constant and $\eta$ is an upper bound on the optimal value $f\left(x^{*}\right)$. It can be shown that for $\eta>f\left(x^{*}\right)$, the minimizer of $\phi(x ; \eta)$ coincides with the minimizer $x(\mu)$ of $P(x ; \mu)$ for some value of $\mu$. The algorithms apply Newton's method to find approximate minima of $\phi(x ; \eta)$ for successivly decreasing values of $\eta$. Variants of this approach are analyzed by Jarre [16] and Den Hertog, Roos, and Terlaky [6], while in [7] the latter authors outline an extension to the logbarrier function $P(x ; \mu)$ of this paper.

Relevant results include those of Jarre [16, Lemma 4] and Nesterov and Nemirovskii [23, Theorem 2.2.3], who prove that Newton's method applied to $\phi(x ; \eta)$ generates a sequence of steps $s_{1}, s_{2}, \ldots$ and iterates $w_{1}, w_{2}, \ldots$ (with $\left.w_{j+1}=w_{j}+s_{j}\right)$ such that the quantity

$$
\left[s_{j}^{T} \phi_{x x}\left(w_{j} ; \eta\right) s_{j}\right]^{1 / 2}=\left[\phi_{x}\left(w_{j} ; \eta\right)^{T} \phi_{x x}\left(w_{j} ; \eta\right)^{-1} \phi_{x}\left(w_{j} ; \eta\right)\right]^{1 / 2}
$$

converges quadratically to zero. This behavior requires the initial estimate $w_{1}$ to be close enough to the minimizer of $\phi(x ; \eta)$ that the bound

$$
\left[\phi_{x}\left(w_{j} ; \eta\right)^{T} \phi_{x x}\left(w_{j} ; \eta\right)^{-1} \phi_{x}\left(w_{j} ; \eta\right)\right]^{1 / 2}<\beta
$$


is satisfied, for some constant $\beta$ that does not depend on $\eta$. Without embarking on a detailed discussion of the relationship of these results to Section 4, we note that near a solution $x^{*}$, the eigenvalues of $\phi_{x x}(x ; \eta)$ can be partitioned into two size classes, in a similar way to those of $P_{x x}(x ; \mu)$, and that weighting of the norm by $\phi_{x x}(w ; \eta)$ has a similar purpose to the partitioning into subspaces spanned by to the orthonormal matrices $\hat{U}$ and $\tilde{U}$ that we use in Section 4 . The neighborhood defined by (95) has a similar extent in the range space of $\hat{U}$ (the active constraint range space) as does our neighborhood (19), for values of $\sigma$ close to 1. However, the results for analytic center methods require the objective and constraint functions to satisfy scaled Lipschitz or self-concordance conditions, whereas this paper assumes the typical smoothness and second-order sufficient conditions that are used in most local analyses of nonlinear programming algorithms. Moreover, the literature on analytic center methods does not, to the best of our knowledge, address the possibility of superlinear convergence.

\section{Acknowledgments}

I am grateful to Florian Jarre for his advice and many helpful comments on earlier drafts of this paper. I am also grateful to the referees and associate editor for their criticisms of an earlier draft, which prompted a major overhaul of the approach and the analysis.

This research was supported by the Mathematics, Information, and Computational Sciences Division subprogram of the Office of Advanced Scientific Computing Research, U.S. Department of Energy, under Contract W-31-109Eng-38.

\section{References}

1. A. Benchakroun, J.-P. Dussault, and A. Mansouri. A two parameter mixed interior-exterior penalty algorithm. ZOR - Mathematical Methods of Operations Research, 41:25-55, 1995.

2. C. Broyden and N. Attia. A smooth sequential penalty function method for solving nonlinear programming problems. In P. Thoft-Christenses, editor, System Modeling and $\mathrm{Op}$ timization, pages 237-245. Springer-Verlag, Berlin, 1983.

3. Richard H. Byrd, MaryBeth Hribar, and Jorge Nocedal. An interior point algorithm for large scale nonlinear programming. OTC Technical Report 97/05, Optimization Technology Center, July 1997.

4. A. R. Conn, N. I. M. Gould, D. Orban, and Ph. L. Toint. A primal-dual trust-region algorithm for minimizing a non-convex function subject to general inequality and linear equality constraints. Technical Report RAL-TR-1999-054, Computational Sciences and Engineering Department, Atlas Center, Rutherford Appleton Laboratory, August 1999.

5. A. R. Conn, N. I. M. Gould, and Ph. L. Toint. A note on using alternative secondorder models for the subproblems arising in barrier function methods for minimization. Numerische Mathematik, 68:17-33, 1994.

6. D. den Hertog, C. Roos, and T. Terlaky. A large-step analytic center method for a class of smooth convex programming problems. SIAM Journal on Optimization, 2(1):55-70, 1992.

7. D. den Hertog, C. Roos, and T. Terlaky. On the classical logarithmic barrier function method for a class of smooth convex programming problems. Journal of Optimization Theory and Applications, 73(1):1-25, 1992. 
8. John E. Dennis and Robert B. Schnabel. Numerical Methods for Unconstrained Optimization. Prentice-Hall, Englewood Cliffs, NJ, 1983.

9. J.-P. Dussault. Numerical stability and efficiency of penalty algorithms. SIAM Journal on Numerical Analysis, 32(1):296-317, 1995.

10. A. V. Fiacco and G. P. McCormick. Nonlinear Programming: Sequential Unconstrained Minimization Techniques. Wiley, New York, 1968. Reprinted by SIAM Publications, 1990.

11. R. Fletcher. Practical Methods of Optimization. John Wiley and Sons, New York, second edition, 1987.

12. A. Forsgren and P. E. Gill. Primal-dual interior-point methods for nonconvex nonlinear programming. SIAM Journal on Optimization, 8(4):1132-1152, 1998.

13. David M. Gay, Michael L. Overton, and Margaret H. Wright. A primal-dual interior method for nonconvex nonlinear programming. Technical Report 97-4-08, Computing Sciences Research, Bell Laboratories, Murray Hill, NJ, July 1997.

14. N. I. M. Gould. On the accurate determination of search directions for simple differentiable penalty functions. IMA Journal of Numerical Analysis, 6:357-372, 1986.

15. N. I. M. Gould. On the convergence of a sequential penalty function method for constrained minimization. SIAM Journal on Numerical Analysis, 26(1):107-128, 1989.

16. F. Jarre. Interior-point methods for convex programming. Applied Mathematics and Optimization, 26:287-311, 1992.

17. K. Jittorntrum and M. R. Osborne. Trajectory analysis and extrapolation in barrier function methods. Journal of the Australian Mathematical Society, Series B, 20:352-369, 1978.

18. F. A. Lootsma. Hessian matrices of penalty functions for solving constrained optimization problems. In Philips Research Reports, volume 24, pages 322-331. Philips, Eindhoven, The Netherlands, 1969.

19. W. Murray. Analytical expressions for the eigenvalues and eigenvectors of the Hessian matrices of barrier and penalty functions. Journal of Optimization Theory and Applications, $7: 189-196,1971$.

20. W. Murray and M. H. Wright. Line search procedures for the logarithmic barrier function. SIAM Journal on Optimization, 4:229-246, 1994.

21. S. G. Nash and A. Sofer. A barrier method for large-scale constrained optimization. ORSA Journal on Computing, 5:40-53, 1993.

22. S. G. Nash and A. Sofer. Why extrapolation helps barrier methods. Technical report, Operations Research and Engineering Department, George Mason University, September 1998.

23. Yu. E. Nesterov and A. S. Nemirovskii. Interior Point Polynomial Methods in Convex Programming. SIAM Publications, Philadelphia, 1994.

24. M. H. Wright. Interior methods for constrained optimization. In Acta Numerica 1992, pages 341-407. Cambridge University Press, Cambridge, 1992.

25. M. H. Wright. Some properties of the Hessian of the logarithmic barrier function. Mathematical Programming, 67:265-295, 1994.

26. M. H. Wright. Why a pure primal Newton barrier step may be infeasible. SIAM Journal on Optimization, 5:1-12, 1995.

27. M. H. Wright. Ill-conditioning and computational error in interior methods for nonlinear programming. SIAM Journal on Optimization, 9:84-111, 1998.

28. S. J. Wright. Effects of finite-precision arithmetic on interior-point methods for nonlinear programming. Preprint ANL/MCS-P705-0198, Mathematics and Computer Science Division, Argonne National Laboratory, Argonne, Ill., January 1998.

29. S. J. Wright and F. Jarre. The role of linear objective functions in barrier methods. Mathematical Programming, Series A, 84:357-373, 1998. 